\title{
BLAZAR 3C 454.3 IN OUTBURST AND QUIESCENCE DURING 2005-2007: TWO VARIABLE SYNCHROTRON EMISSION PEAKS
}

\author{
Patrick M. Ogle ${ }^{1}$, Ann E. Wehrle ${ }^{2}$, Thomas BaloneK ${ }^{3}$, and Mark A. Gurwell ${ }^{4}$ \\ ${ }^{1}$ Spitzer Science Center, California Institute of Technology, Mail Code 220-6, Pasadena, CA 91125, USA; ogle@ipac.caltech.edu \\ ${ }^{2}$ Space Science Institute, 4750 Walnut Street, Suite 205, Boulder, CO 80301, USA \\ ${ }^{3}$ Department of Physics and Astronomy, Colgate University, Colgate, NY 13346, USA \\ ${ }^{4}$ MS42, Smithsonian Astrophysical Observatory, 60 Garden Street, Cambridge, MA 02138, USA \\ Received 2010 March 16; accepted 2011 April 19; published 2011 August 3
}

\begin{abstract}
We monitored the flaring blazar 3C 454.3 during 2005 June-July with the Spitzer Infrared Spectrograph (IRS: 15 epochs), Infrared Array Camera (IRAC: 12 epochs), and Multiband Imaging Photometer (MIPS: 2 epochs). We also made Spitzer IRS, IRAC, and MIPS observations from 2006 December to 2007 January when the source was in a low state, the latter simultaneous with a single Chandra X-ray observation. In addition, we present optical and submillimeter (sub-mm) monitoring data. The 2005-2007 period saw three major outbursts. We present evidence that the radio-optical spectral energy distribution (SED) actually consists of two variable synchrotron peaks, the primary at IR and the secondary at sub-mm wavelengths. The lag between the optical and sub-mm outbursts may indicate that these two peaks arise from two distinct regions along the jet separated by a distance of $0.9-3 \mathrm{pc}$. The flux at $5-35 \mu \mathrm{m}$ varied by a factor of 40 and the IR peak varied in frequency from $4 \times 10^{12} \mathrm{~Hz}$ to $4 \times 10^{13} \mathrm{~Hz}$ between the highest and lowest states in 2005 and 2006, respectively. Variability was well correlated across the mid-IR band, with no measurable lag. Flares that doubled in flux occurred on a timescale of $\sim 5$ days, yielding a variability size of $<0.05 \mathrm{pc}$. The IR SED peak moved to higher frequency as a flare brightened, then returned to lower frequency as it decayed. The fractional variability amplitude increased with frequency, which we attribute to decreasing synchrotron self-absorption optical depth. Mid-IR flares may signal the re-energization of a shock that runs into inhomogeneities along the pre-existing jet or in the external medium. The synchrotron peak frequencies during each major outburst may depend upon both the distance from the jet apex and the physical conditions in the shocks. Variation of the Doppler parameter along a curved or helical jet is another possibility. Frequency variability of the IR synchrotron peak may have important consequences for the interpretation of the blazar sequence, and the presence of a secondary peak may give insight into jet structure.
\end{abstract}

Key words: galaxies: jets - quasars: individual (3C 454.3)

Online-only material: color figure, machine-readable tables

\section{INTRODUCTION}

In 2005 May-September, the blazar 3C $454.3(z=0.859)$ underwent the largest radio-optical flare in its recorded history. In 2005 May, it briefly surpassed 3C 273 as the optically brightest quasar in the sky in spite of its much greater distance (Balonek 2005a, 2005b). This flaring event was intensively monitored at all frequencies by observers all over the world, using both ground- and space-based observatories (Villata et al. 2006, 2007; Pian et al. 2006; Fuhrmann et al. 2006; Raiteri et al. 2007). The source has since flared several times with smaller amplitude, offering continuing opportunities to study this spectacular phenomenon, e.g., Raiteri et al. (2008a, 2008b), Villata et al. (2009), Hagen-Thorn et al. (2009), Vercellone et al. (2009), Bonning et al. (2010), Sasada et al. (2010), Smith \& Wehrle (2010), and Larionov et al. (2010). Multiwavelength analyses of flaring since 2005 are presented by Jorstad et al. (2010), Vercellone et al. (2010), Bonning et al. (2009), and Pacciani et al. (2010).

Blazars, including flat-spectrum radio-loud quasars and $\mathrm{BL}$ Lac objects, are characterized by strong variability and high polarization. According to active galaxy unification models, they are viewed within $\sim 20^{\circ}$ of the radio jet axis. The spectral energy distribution (SED) is dominated by relativistically boosted emission from the core of the jet. The core is strongly variable at all frequencies, owing to rapid movement and changes in the jet on subparsec scales. Very long baseline interferometry (VLBI) typically shows jet components with apparently superluminal motion at milliarcsecond resolution, demonstrating the importance of relativistic effects.

The SEDs of blazars are typically characterized by two major bumps, one which peaks in the radio through X-ray bands, and the other which peaks in $\mathrm{X}-\gamma$-ray bands. The low-frequency bump is highly polarized, indicating synchrotron emission from relativistic electrons in a magnetized jet. The high-frequency bump may be produced by either inverse Compton scattering of photons by relativistic electrons in leptonic jet models or protonsynchrotron emission in lepto-hadronic models (Böttcher 2010, and references therein). In inverse Compton models the source of photons is debated, ranging from jet electron-synchrotron emission to external photon fields (e.g., Wehrle et al. 1998; Finke \& Dermer 2010).

In some blazars, a significant fraction of the optical-UV thermal emission as well as broad optical-UV emission lines may arise from an accretion disk (Smith et al. 1988). See, for example, Ghisellini \& Tavecchio (2009) and references therein, for a review of models and their application to the class of flat-spectrum radio quasars (FSRQs).

Theoretical unifying schemes for $\gamma$-ray bright blazars have been proposed: the more luminous the blazar, the lower the peak frequencies of the synchrotron and $\gamma$-ray bumps (Fossati et al. 1998; Ghisellini et al. 1998). For inverse Compton models, 
the downshift in peak frequency can be caused by increased Compton cooling at higher radiation densities. In the externalCompton (EC) model (e.g., Sikora et al. 1994), the higher radiation density comes from scattered UV-optical accretion disk and broad-line region (BLR) photons, and IR photons from the dusty torus. Hence, luminous FSRQ SEDs peak (on average) at lower frequencies than do those of BL Lac objects, which have weaker (if any) accretion disk, BLR, and torus emission. Similarly, we might expect accretion disk, BLR, and torus luminosity variations in a single object to be accompanied by changes in the frequency of the synchrotron peak.

However, cooling may be counterbalanced by re-acceleration of high-energy electrons in jet shocks. As a disturbance travels down the jet, it may run into inhomogeneities internal to or external to the jet. At such locations, the bulk relativistic kinetic energy and energy in the electromagnetic field is dissipated, heating the electrons, which emit copious synchrotron photons as they gyrate about the magnetic field lines. The increased number of high-energy electrons will shift the synchrotron peak to higher frequencies. Thus, the peak synchrotron frequency is determined by a balance between shock heating and cooling, and should vary as jet shock components evolve. An exact correspondence between accretion luminosity and peak synchrotron frequency is therefore not expected.

The IR is a crucial band since it covers the primary synchrotron emission peak of FSRQs, which is determined by the relativistic electron and magnetic field energy densities, and may provide seed photons for a Compton $\gamma$-ray bump. In addition, while the base of the jet is optically thick at radio frequencies, it becomes optically thin at mid-IR through UV frequencies. These frequencies are less subject to optical depth effects than radio observations and can be used to probe short variability timescales which arise close to the origin of the jet. If flaring episodes are caused by shocks (Marscher \& Gear 1985), then flares should occur simultaneously across the IR through UV wave bands (Lainela 1994; Savolainen et al. 2002). Therefore, IR spectral variability should reflect changes in the energy distribution of relativistic electrons. Variability on day to month timescales can be used to study the evolution of the shock as it propagates along the jet and through the environment of the active galactic nucleus (AGN).

The blazar 3C 454.3 has undergone a number of flaring episodes, with bright radio outbursts occurring roughly once every six years (Ciaramella et al. 2004). It has a one-sided milliarcsecond scale radio jet (Pearson et al. 1980; Cawthorne \& Gabuzda 1996; Marscher et al. 2002; Scott et al. 2004; Jorstad et al. 2005). VLBI monitoring observations reveal a series of jet components, including stationary components and components with apparent superluminal motion of up to $16 \mathrm{~h}^{-1} c$ (PaulinyToth et al. 1987; Gomez et al. 1999; Jorstad et al. 2001, 2005, 2010; Kellerman et al. 2004). Jorstad et al. (2010) found that a superluminal knot (K1) was ejected from the core in mid-2005 (JD $2452553+/-29$ days; $2005.5+/-0.08$ ), and derived the following parameters: apparent speed $\beta_{\text {app }}=4.9 \pm 0.9$, Lorentz factor $\Gamma_{j}=12.3$, Doppler factor $\delta=23.4$, and angle to line of sight $\Theta_{o}=1.0$. This knot is very likely associated with the 2005 May optical-IR outburst, so we adopt these values in our discussion of the synchrotron emission regions.

Historically, the synchrotron emission from 3C 454.3 typically peaks in $v F_{v}$ in the far-IR $(\sim 100 \mu \mathrm{m})$. IR emission observed by the Infrared Space Observatory (ISO) had a flux of $34 \mathrm{mJy}$ at $12.8 \mu \mathrm{m}$ (Haas et al. 2004). Excess emission in the SED at $60 \mu \mathrm{m}$ was attributed to thermal emission from $100 \mathrm{~K}$ dust by these authors, but we will present evidence that this emission is highly variable.

The greatest amount of energy is emitted in the $\gamma$-ray bump, which peaks at $\sim 10 \mathrm{MeV}$, but extends down to soft $\mathrm{X}$-ray energies. The X-ray emission observed with Beppo-SAX was characterized by a $0.5-200 \mathrm{keV}$ power law with spectral index $\alpha=-2.3$ (Tavecchio et al. 2002). Gamma-ray emission was observed at energies of $0.05-4 \mathrm{GeV}$ by the EGRET, OSSE, and COMPTEL instruments on the Compton Gamma-Ray Observatory (Hartman et al. 1993; McNaron-Brown et al. 1995; Zhang et al. 2005). Gamma-ray detections after the data in this paper were obtained include those by AGILE (Vercellone et al. 2008, 2009) in 2007, by the Large Area Telescope on Fermi in 2008 (Abdo et al. 2009), and by AGILE in 2008-2009 (Donnarumma et al. 2009a); see also the review by Vercellone et al. (2010). Gamma-ray flares in 2007-2010 are investigated by Bonnoli et al. (2011), Foschini et al. (2010), Ackermann et al. (2010), Striani et al. (2010), and Abdo et al. (2009), and an upper limit to very high energy ( $\mathrm{TeV}$ ) flux is given by Anderhub et al. (2009).

We were awarded director's discretionary time to observe 3C 454.3 daily with the Spitzer Infrared Spectrograph (IRS), the Infrared Array Camera (IRAC), and the Multiband Imaging Photometer (MIPS) over a period of four weeks in 2005 July, during normally scheduled instrument campaigns. We also took coordinated Spitzer MIPS, IRS, IRAC, and Chandra High Energy Transmission Grating (HETG) observations in 2006 December to 2007 January. We obtained observations from long-term optical monitoring programs at Colgate University and Palomar Observatory and from calibrator monitoring at the Submillimeter Array (SMA). In this paper, we present these data and discuss variability and the nature of synchrotron emission flares from the blazar 3C 454.3.

Angular sizes and luminosities in this paper are calculated assuming a cosmology with Hubble constant $H_{0}=70 \mathrm{~km} \mathrm{~s}^{-1}$ $\mathrm{Mpc}^{-1}$, matter density parameter $\Omega=0.3$, and dark energy density $\Omega_{\Lambda}=0.7$.

\section{A BRIEF HISTORY OF 3C 454.3 IN 2005-2008}

Observations that were made during the same time period as those reported in this paper included several large multiwavelength campaigns, summarized here. Following the alert of an optical flare in 3C 454.3 by Balonek (2005a) and Balonek (2005b), Fuhrmann et al. (2006) obtained near-IR/optical photometry starting two days later. Villata et al. (2006) made the first report of the WEBT multiwavelength campaigns, including Target of Opportunity pointings by Chandra and the International Gamma-Ray Astrophysics Laboratory (INTEGRAL), that followed the discovery of the 2005 May optical flare. Raiteri et al. (2007) reported the detection of the "small and big blue bumps" during the low state in 2006. Villata et al. (2007) derived the delay between centimeter-band radio and optical light curves during 2005-2006. Giommi et al. (2006) observed 3C 454.3 with the robotic Rapid Eye Mount optical/near-infrared telescope and with the Swift satellite in 2005 April-May. Raiteri et al. (2008b) presented multifrequency observations by the WEBT and XMMNewton in 2007-2008, overlapping with AGILE's 2007 November observations. They also used $1.3 \mathrm{~mm}$ monitoring data from the SMA, some of which are also used in our paper, over the time period 2005-2008 (see their Figure 4) as well as $1.3 \mathrm{~mm}$ data from Pico Veleta, Spain. X-ray data from Swift, Chandra, XMM-Newton, and INTEGRAL data from the 2005-2007 period have been presented by Giommi et al. (2006), Villata 
et al. (2006), Raiteri et al. (2007), and Pian et al. (2006). We describe the results of these papers as follows.

1. Fuhrmann et al. (2006) observed 3C 454.3 with the Automatic Imaging Telescope of Perugia University Observatory and the Rapid Eye Mount telescope in Chile, obtaining $V$-, $R$-, $I$-, and $H$-band photometry. The spectral index over these bands showed no strong significant changes.

2. Villata et al. (2006) presented results up through 2005 September, finding that the source was redder when brighter. A millimeter outburst occurred in 2005 June-July, followed months later by the $37-43 \mathrm{GHz}$ peak. Chandra and INTEGRAL X-ray observations in 2005 May showed unusually high fluxes.

3. Giommi et al. (2006) observed 3C 454.3 with the robotic Rapid Eye Mount optical/near-infrared telescope and with the Swift satellite in 2005 April-May. They found that the optical and ultraviolet flux doubled within a single $1.5 \times 10^{5} \mathrm{~s}$ exposure and the X-Ray Telescope $(2-10 \mathrm{keV})$ $\mathrm{X}$-ray flux varied little during the same time. However, on timescales of a few days, the Burst Alert Telescope $(15-150 \mathrm{keV}) \mathrm{X}$-ray flux varied by more than a factor of three; in contrast, the average level of the optical-ultraviolet flux was approximately constant between the two UVOT observations of 2005 April 24 and May 17.

4. Villata et al. (2007) reported multiwavelength observations during 2005-2006. Their analysis suggested that the big radio flare $(43-37 \mathrm{GHz})$ in early 2006 was associated with a minor optical flare in 2005 October-November, not with the 2005 May major optical flare. A combination of disturbances traveling down the jet and changes of viewing angles of different emitting regions, with concomitant changes in Doppler boosting, were found to explain the radio delays with respect to the optical emission.

5. Raiteri et al. (2007) detected the "small and big blue bumps" during the low state in 2006. Observations from the $K$ band through ultraviolet showed an upturn at the infrared end, a small peak in optical band $(V)$, and an upturn in ultraviolet from the $U$ band to $U V W 1$ and $U V M 2$. The infrared emission was probably variable synchrotron, complicated by a non-variable $\mathrm{H}$-alpha line (reported by Raiteri et al. 2008 b via spectroscopy in the $J$ band). The "small blue bump" is probably a blend of iron lines, $\mathrm{Mg}$ II lines, and Balmer continuum from the BLR. Bonnoli et al. (2011) reported the Galaxy Evolution Explorer (GALEX) detection of strong Ly $\alpha$ emission from the BLR in observations made between 2008 September 30 and October 2. Ly $\alpha$ is observed at $2260 \AA$, within the Swift ultraviolet $U V M 2$ filter, and overlapping slightly with the $U V W 1$ and $U V W 2$ filters (Bonnoli et al. 2011, Figure 3). This emission line contributes to the UV upturn in the UVOT SED which is especially visible when the source is faint and adds to the contribution from the accretion disk identified by Raiteri et al. (2007). All of these emission components contributed during various activity levels. The underlying accretion disk and broad-line contributions were visible most clearly when the synchrotron emission was at low ebb.

The Raiteri et al. (2007) X-ray spectra could be fitted with a power law, but seemed to require extra $N_{\mathrm{H}}$ absorption some of the time. Alternatively, there may have been spectral changes in the soft X-ray range.

6. Raiteri et al. (2008b) presented multifrequency observations by the WEBT and XMM-Newton in 2007-2008, overlapping with AGILE's 2007 November observations. They
Table 1

Submillimeter Array Photometry

\begin{tabular}{lcrcccrc}
\hline \hline Year $^{\mathrm{a}}$ & Month & Day & UT & MJD & $\begin{array}{c}\text { Freq } \\
(\mathrm{GHz})\end{array}$ & $\begin{array}{r}\text { Flux } \\
(\mathrm{Jy})\end{array}$ & $\begin{array}{r}\text { Error } \\
(\mathrm{Jy})\end{array}$ \\
\hline 2005 & 01 & 13 & $02: 57$ & 53383.121 & 225.6 & 9.7 & 0.5 \\
2005 & 04 & 26 & $16: 10$ & 53486.672 & 340.8 & 18.0 & 1.1 \\
2005 & 04 & 27 & $15: 57$ & 53487.664 & 340.8 & 22.2 & 1.2 \\
2005 & 05 & 2 & $05: 36$ & 53492.234 & 342.5 & 25.0 & 1.4 \\
2005 & 05 & 6 & $05: 42$ & 53496.238 & 225.5 & 25.9 & 1.3 \\
2005 & 05 & 7 & $07: 08$ & 53497.297 & 340.9 & 23.9 & 1.2 \\
2005 & 05 & 8 & $07: 16$ & 53498.305 & 340.8 & 24.1 & 1.2 \\
2005 & 05 & 9 & $05: 31$ & 53499.230 & 340.9 & 24.0 & 1.3 \\
2005 & 05 & 10 & $04: 00$ & 53500.168 & 340.9 & 25.1 & 1.3 \\
2005 & 05 & 16 & $06: 21$ & 53506.266 & 336.6 & 24.2 & 1.4 \\
\hline
\end{tabular}

Note. ${ }^{a}$ UT date.

(This table is available in its entirety in a machine-readable form in the online journal. A portion is shown here for guidance regarding its form and content.)

also used $1.3 \mathrm{~mm}$ monitoring data from the SMA, some of which is also used in our paper, over the time period 2005-2008 (see their Figure 4) as well as $1.3 \mathrm{~mm}$ data from Pico Veleta, Spain. Correlation of the WEBT $R$-band optical data with the SMA and Pico Veleta data showed that the millimeter fluxes lagged the optical fluxes by 40-80 days, with Discrete Correlation Function (DCF) maximum signal at 65 days. Taking only non-outburst data for the correlation yielded a DCF maximum at 20 days. They suggested that the "jet regions emitting the optical and millimeter radiations are now better aligned than in the past, and/or that the opacity in the jet has decreased, allowing the release of millimeter radiation closer to the optically emitting zone." We return to this correlation later in our paper. Several multiwavelength SEDs during 2005-2007 are shown and analyzed. From the X-ray-optical correlation, they found that the X-ray, near-infrared, and optical emission would be produced in the same spatial region.

\section{OBSERVATIONS}

\subsection{Submillimeter Array Observations}

The SMA is an eight-element radio interferometer located atop Mauna Kea, Hawaii, which operates in the $1.3 \mathrm{~mm}, 850 \mu \mathrm{m}$, and $450 \mu \mathrm{m}$ atmospheric windows (Ho et al. 2004). In the $1.3 \mathrm{~mm}$ and $850 \mu \mathrm{m}$ windows, quasars are utilized as amplitude and phase gain calibrator sources during routine observing, and the flux density scale is derived through observations of standards in each session, typically solar system sources (with Uranus, Neptune, Titan, Ganymede, and Callisto being the most used). The SMA supports a program to calibrate quasar flux densities using both routine science and dedicated calibration observations, and provide their histories to SMA users and the wider astronomical community. The Submillimeter Calibrator List, containing the flux measurement histories of several hundred quasars, can be found in the Tools section of the SMA Observer Center, ${ }^{5}$ with further details provided by Gurwell et al. (2007). Data for 2005-2007 are presented in Table 1 and Figure 1 for the $850 \mu \mathrm{m}$ and $1 \mathrm{~mm}$ bands.

\subsubsection{Submillimeter Flares}

The submillimeter (sub-mm) data, shown in Figure 1(a), show a huge flare peaking at 42.7 Jy on 2005 June 24 and

\footnotetext{
5 http://sma1.sma.hawaii.edu
} 

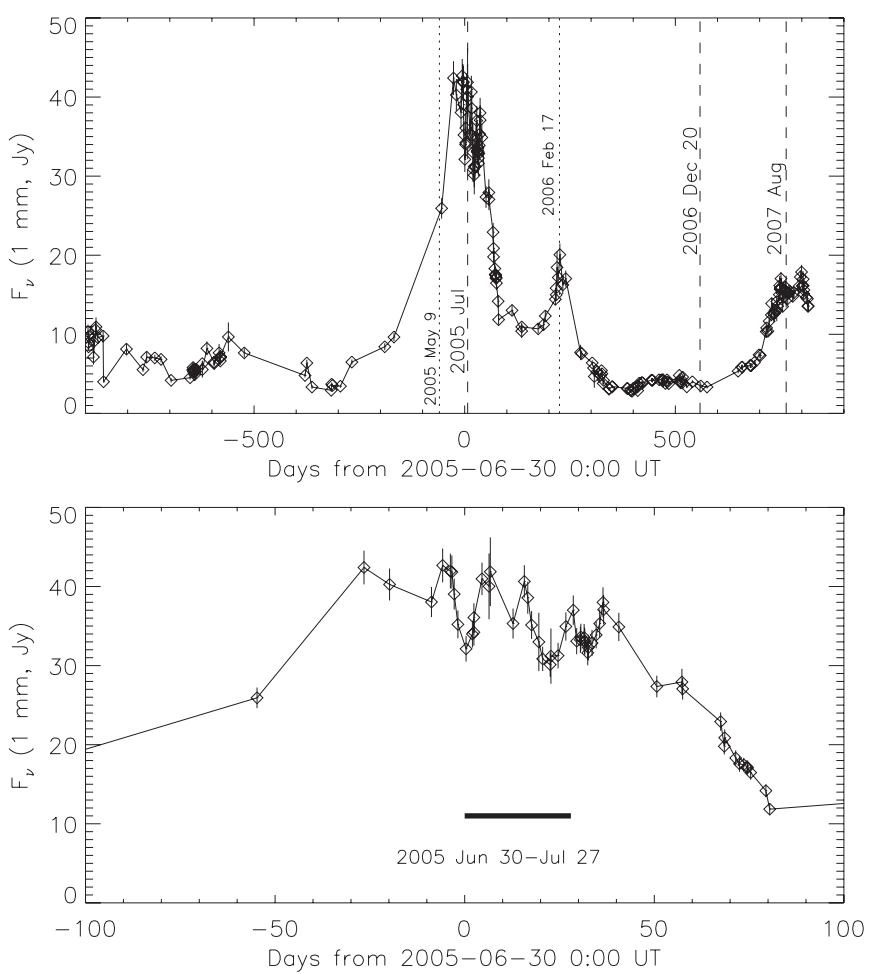

Figure 1. Radio $(1.3 \mathrm{~mm}=230 \mathrm{GHz})$ variability of 3C 454.3. Top: light curve from 2002-2007. The date of the earliest WEBT observation of the 2005 May optical outburst is indicated by the dotted line. The dates of our Spitzer observations are indicated by dashed lines. Bottom: 2005 outburst with the period of the first Spitzer monitoring campaign indicated by the solid bar.

a smaller flare of $20 \mathrm{Jy}$ in 2006 February. Figure 1(b) shows the extraordinary flare in 2005 and a surprisingly large amount of substructure during the two-month period around the peak, including five dates on which the flux exceeded $40 \mathrm{Jy}$. We think the substructure is real rather than random noise because the variability correlates well between the 230 and $345 \mathrm{GHz}$ band measurements with several measurements in a row showing consistent increasing or decreasing flux density. We cannot completely exclude systematic error, for example, observer bias in knowing what the preceding measurement value was.

The SMA is sensitive to only a single polarization which also rotates on the sky as a function of elevation. Anecdotal reports by other observers indicated that 3C 454.3 was not polarized by more than a couple of percent. Given the complex rotation relative to the source, it is unlikely that any significant structure in the time variability would be related to changes in the polarization angle, but it cannot be excluded without considerable extra work beyond the scope of this paper.

For about $98 \%$ of the measurements, the frequency quoted is in fact the local oscillator (LO) frequency, and the flux measurement is the average of the lower and upper sideband values (in the heterodyne mixing, the sky frequencies are mixed down to the more manageable IF frequency range of 4-6 GHz, but we accept both sidebands, e.g., LO +/- (4-6 GHz). The use of interferometry and the complex correlator allows us to unambiguously separate the sidebands, so we really get two flux measurements, separated by $10 \mathrm{GHz}$. The SMA 3C 454.3 data yield a spectral index that typically lies near $v^{-0.5}$, thus we would expect only a $2 \%$ drop in flux from 220 to $230 \mathrm{GHz}$ (1.3 mm band), and only $1.5 \%$ from 335 to $345 \mathrm{GHz}(850 \mu \mathrm{m}$ band). The peak substructure in the sub-mm flare is definitely not caused simply by changes in the frequency of observation.
Table 2

Spitzer Infrared IRAC and MIPS Observations of 3C 454.3

\begin{tabular}{cccccc}
\hline \hline Year $^{\mathrm{a}}$ & Month & Day & Instrument & Wave Band $(\mu \mathrm{m})$ & Integration Time $^{\mathrm{b}}$ \\
\hline 2005 & 7 & $14-26$ & IRAC & $3.6,4.5,5.8,8$ & $1 \times 32 \mathrm{~s}$ \\
2005 & 7 & $27\left(\right.$ twice $\left.^{\mathrm{c}}\right)$ & MIPS & $24,70,160$ & $(2,2,4) \times 3 \mathrm{~s}$ \\
2006 & 12 & 25 & IRAC & $3.6,4.5,5.8,8$ & $1 \times 32 \mathrm{~s}$ \\
2007 & 1 & 1 & MIPS & 24,70 & $2 \times 3 \mathrm{~s}$ \\
\hline
\end{tabular}

Notes.

${ }^{\text {a }}$ UT date.

b Number of exposures or cycles $\times$ exposure time (s).

${ }^{c}$ Observed at 01:48 UT and 15:11 UT.

Table 3

Spitzer IRS Observations of 3C 454.3

\begin{tabular}{lccccccc}
\hline \hline Epochs & Year $^{\mathrm{a}}$ & Month & Day & SL $^{\mathrm{b}}$ & LL & SH & LH \\
\hline 1 & 2005 & 6 & 30 & $4 \times 60$ & $2 \times 120$ & $4 \times 120$ & $4 \times 60$ \\
$2-14$ & 2005 & 7 & $1-12$ & $4 \times 60$ & $2 \times 120$ & 0 & 0 \\
15 & 2005 & 7 & 13 & $4 \times 60$ & $2 \times 120$ & $4 \times 120$ & $4 \times 60$ \\
16 & 2006 & 12 & 20 & $16 \times 14$ & $24 \times 14$ & 0 & 0 \\
\hline
\end{tabular}

Notes.

${ }^{\text {a }}$ UT date.

b Number of exposures $\times$ exposure time (s).

\subsection{Spitzer Observations}

We observed 3C 454.3 with Spitzer IRS, IRAC, and MIPS at several epochs during 2005-2007 (Tables 2 and 3). Note that the three instruments were operated separately (according to their regularly scheduled campaigns of typical length 1-2 weeks) so we do not have simultaneous coverage in the respective IR bands. First, we observed the source once per day from 2005 June 30 to 2005 July 27 to track mid-IR spectral variability following the 2005 May outburst. We conducted a series of 14 daily IRS observations between 2005 June 30 and July 12, followed by 12 daily IRAC observations between July 14 and July 26, followed by two MIPS observations on July 27, the last day of the Spitzer visibility window. We observed the source again on 2006 December 20 to 2007 January 2, the first of two coordinated Spitzer and Chandra observation sequences (the second is reported by A. Wehrle et al. 2011, in preparation). We made an observation with IRS on 2006 December 20, with IRAC on 2006 December 25, and with MIPS on 2007 January 1, the latter simultaneously with Chandra as part of a program unrelated to the flare. All IRS observations were made in staring mode, and IRAC and MIPS images were taken in mapping modes.

\subsubsection{IRAC}

The IRAC images (Figure 2) on 2005 July 17 (bright state) and 2006 December 25 (faint state) show the unresolved quasar in a field of stars and galaxies. The Spitzer Science Center (SSC) pipeline mosaic data (version 14.0.0) were used for aperture photometry carried out with SSC APEX software package in beta release which was the only Spitzer software available for photometry when we began this work. The apertures used were $2,4,5$, and 5 pixels in radius ( 1 pixel $=1.2$ arcsec $)$ at 3.6, 4.5, 5.8, and $8.0 \mu \mathrm{m}$, and corrected using the values given in Table 5.7, $\mathrm{p}$. 53 of the IRAC Data Handbook, version 3.0. We cross-checked the computed quasar fluxes for a range of apertures with those of a nearby star of roughly comparable brightness; the errors are consistent with the $1.5 \%$ systematic errors plus $3 \%$ absolute 


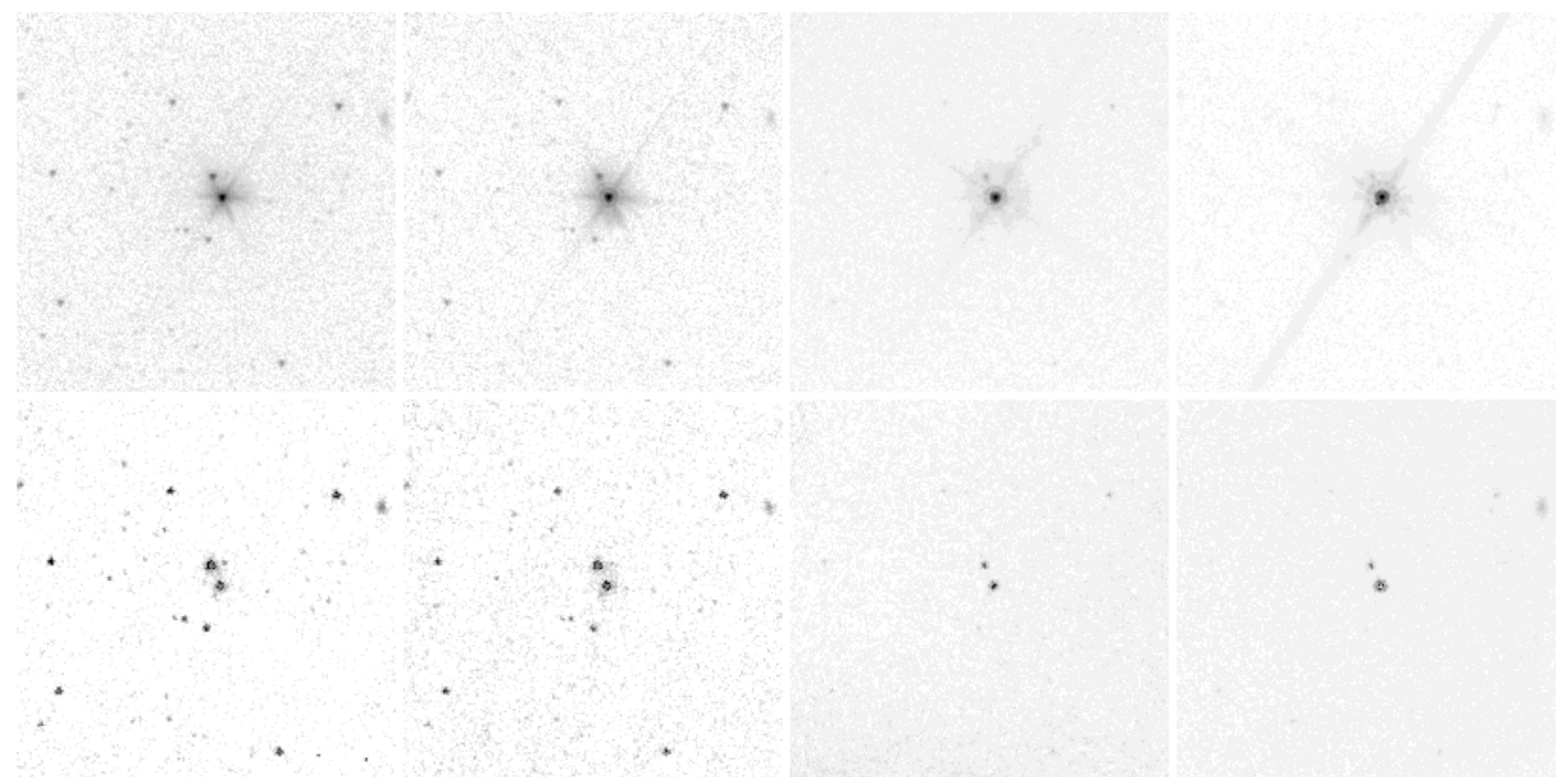

Figure 2. Spitzer IRAC images at 3.6, 4.5, 5.8, and $8 \mu \mathrm{m}$ during the bright state on 2005 July 17 (top four panels) and during the faint state on 2006 December 25 (bottom four panels). Only the central $8 \times 8$ arcmin of each image are shown (north up, east left). The transfer functions of each panel have been chosen individually to show the most detail.

Table 4

Spitzer Infrared IRAC Photometry of 3C 454.3

\begin{tabular}{ccccccccc}
\hline \hline Year $^{\mathrm{a}}$ & Month & Day & UT & JD & $S_{3.6}(\mathrm{Jy})^{\mathrm{b}}$ & $S_{4.5}(\mathrm{Jyy})^{\mathrm{c}}$ & $S_{5.8(\mathrm{Jy})}{ }^{\mathrm{d}}$ & $S_{8.0}(\mathrm{Jy})^{\mathrm{e}}$ \\
\hline 2005 & 7 & 14 & $19: 41$ & 2453566.32014 & 0.172 & 0.247 & 0.37 & 0.616 \\
2005 & 7 & 15 & $17: 52$ & 2453567.24444 & 0.201 & 0.292 & 0.445 & 0.765 \\
2005 & 7 & 16 & $19: 41$ & 2453568.32014 & 0.197 & 0.282 & 0.426 & 0.723 \\
2005 & 7 & 17 & $19: 08$ & 2453569.29722 & 0.239 & 0.329 & 0.481 & 0.774 \\
2005 & 7 & 18 & $21: 03$ & 2453570.37708 & 0.206 & 0.284 & 0.417 & 0.664 \\
2005 & 7 & 19 & $21: 17$ & 2453571.38681 & 0.185 & 0.258 & 0.382 & 0.610 \\
2005 & 7 & 20 & $20: 08$ & 2453572.33889 & 0.163 & 0.228 & 0.335 & 0.533 \\
2005 & 7 & 21 & $20: 51$ & 2453573.36875 & 0.133 & 0.187 & 0.277 & 0.443 \\
2005 & 7 & 22 & $18: 49$ & 2453574.28403 & 0.102 & 0.145 & 0.216 & 0.347 \\
2005 & 7 & 23 & $20: 29$ & 2453575.35347 & 0.092 & 0.128 & 0.187 & 0.293 \\
2005 & 7 & 24 & $08: 28$ & 2453575.85278 & 0.097 & 0.135 & 0.198 & 0.309 \\
2005 & 7 & 25 & $19: 49$ & 2453577.32569 & 0.113 & 0.156 & 0.226 & 0.349 \\
2006 & 12 & 25 & $06: 23$ & 2454081.76597 & $5.65 \mathrm{E}-3$ & $7.30 \mathrm{E}-3$ & $9.10 \mathrm{E}-3$ & $14.2 \mathrm{E}-3$ \\
\hline
\end{tabular}

Notes.

${ }^{\text {a }}$ UT date.

${ }^{\mathrm{b}}$ Flux at $3.6 \mu \mathrm{m}$; error is $5 \%$.

${ }^{\mathrm{c}}$ Flux at $4.5 \mu \mathrm{m}$; error is $5 \%$.

${ }^{\mathrm{d}}$ Flux at $5.8 \mu \mathrm{m}$; error is $5 \%$.

${ }^{\mathrm{e}}$ Flux at $8.0 \mu \mathrm{m}$; error is $5 \%$.

calibration uncertainty of the IRAC (Reach et al. 2005). Despite the unpredictable brightness of the source, none of the IRAC images were saturated. Following the release of a new version of IPAC's Skyview software (courtesy of B. Hartley), we used it to reduce the images independently. The APEX and Skyview results agree to within $2 \%$, except on 2005 July 14, where the APEX value exceeds the Skyview value by $10 \%$ at $3.6 \mu \mathrm{m}$. The Skyview fluxes at 3.6, 4.5, 5.8, and $8 \mu \mathrm{m}$ are listed in Table 4.

The faint state 3.6 and $4.5 \mu \mathrm{m}$ IRAC images show a $10 \sigma$ feature about 6 " from the quasar at position angle (P.A.) $\sim 150 \mathrm{deg}$. This feature appears on the opposite side from the $8^{\prime \prime}$ radio jet at P.A. -45 deg (e.g., Cooper et al. 2007) and its associated optical emission (Cheung et al. 2005). The feature was present in all individual frames when the source was faint, including those from another of our Spitzer programs. It was not associated with typical IRAC instrumental artifacts (J. Surace 2009, private communication). Working with Marco Chiaberge, we pulled archival Advanced Camera for Surveys Hubble images (PI: F. Tavecchio) of the source during a faint episode in 2004 August, and found a plausible identification of the IRAC feature with two galaxies, conflated by the much larger IRAC pointspread function (PSF). The galaxies' brightness and colors indicated that they could be located at roughly the same distance as 3C 454.3 (W. C. Keel 2009, private communication). Figure 3 shows the IRAC and Hubble images side by side. There is no 


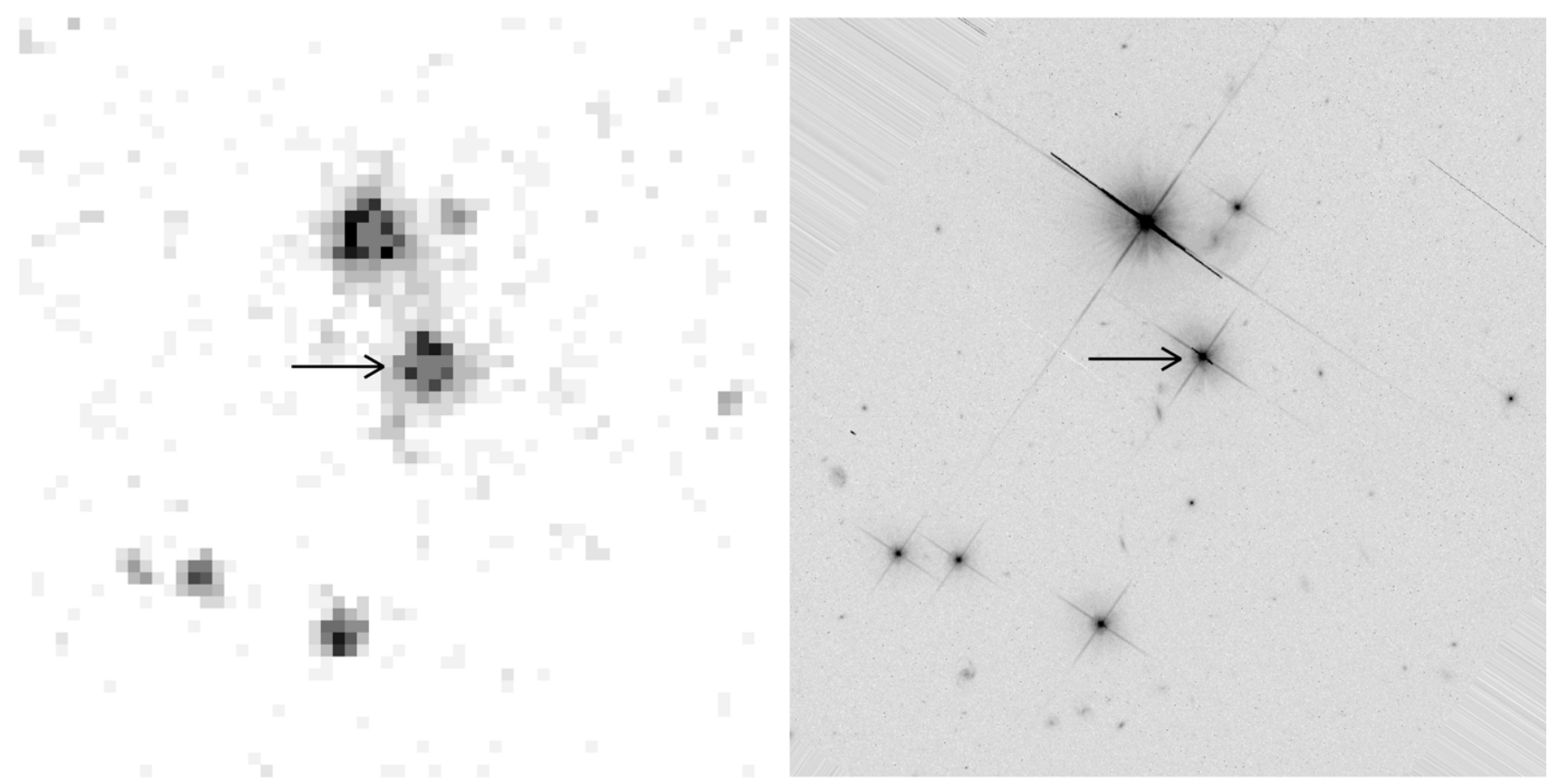

Figure 3. Left: Spitzer IRAC image at $3.6 \mu \mathrm{m}$ during the faint state on 2006 December 25. Right: Hubble image in the F814 filter (2004 August 4, provided by M. Chiaberge). Only the central $1.24 \times 1.24$ arcmin of each image are shown (north up, east left). Arrows mark the position of $3 \mathrm{C} 454.3$ in the two images. The extension to the SE of 3C 454.3 in the IRAC image corresponds to two faint galaxies in the Hubble image.

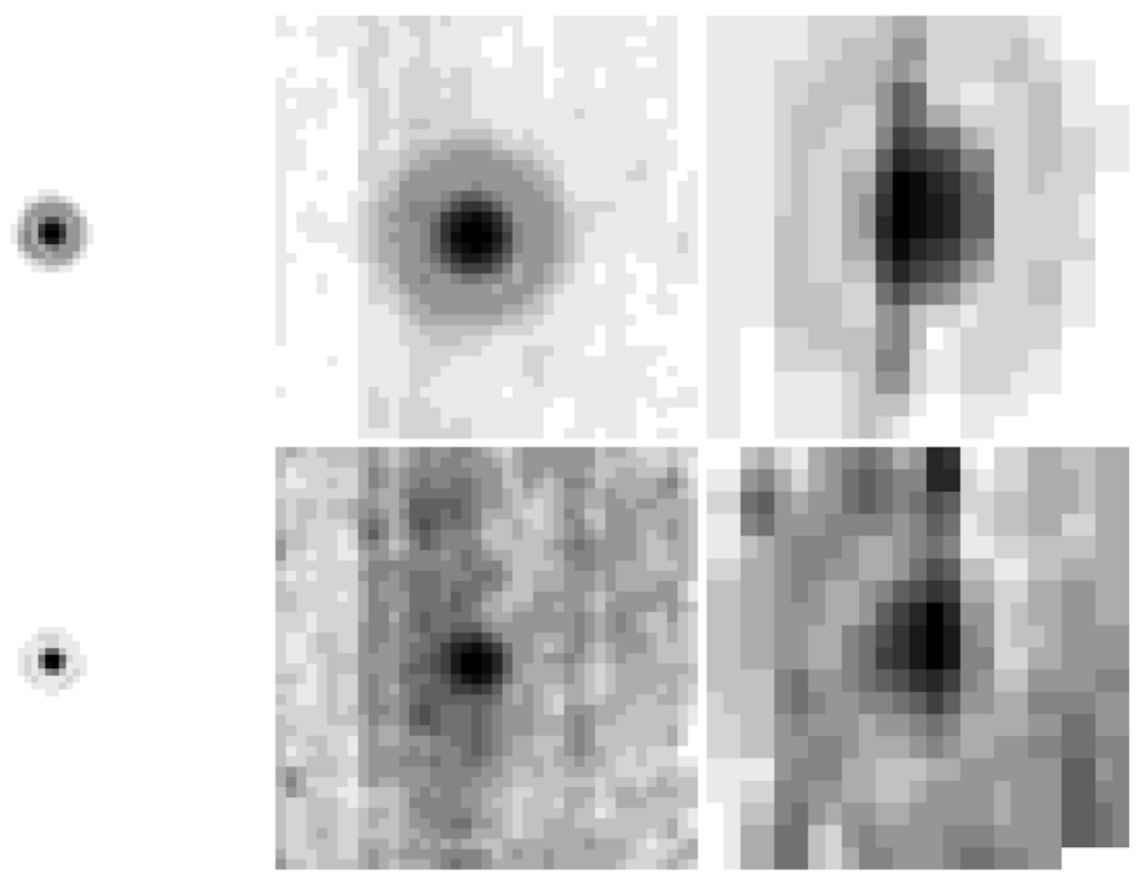

Figure 4. Spitzer MIPS images at $24 \mu \mathrm{m}$ (left), $70 \mu \mathrm{m}$ (middle), and $160 \mu \mathrm{m}$ (right) during the bright state on 2005 July 27 (top) and during the faint state on 2007 January 1 (bottom). Only the centers of each image are shown (in detector coordinates). The transfer functions of each panel have been chosen individually to bring out PSF details.

obvious source of $3.6 \mu \mathrm{m}$ emission corresponding to the jet-side optical hot spot (Cheung et al. 2005), but this in principle may be detected with a deeper image taken when $3 \mathrm{C} 454.3$ is in a faint state.

\subsubsection{MIPS}

The MIPS images (Figure 4), taken when the source was bright on 2005 July 27 at 01:48 UT and 15:11 UT, and when the source was faint on 2007 January 1, show the unresolved quasar. In the full $24 \mu \mathrm{m}$ images, the bright quasar and a few faint galaxies were visible. At $70 \mu \mathrm{m}$, the quasar was isolated on fairly smooth background. The first Airy ring is clearly visible in each image. In the 2005 July 27 data sets, evidence of "soft saturation" was found in the $160 \mu \mathrm{m}$ data (the pipeline images showed a doughnut-like structure instead of a smooth peak). Special MIPS custom processing on the Basic Calibrated Data 


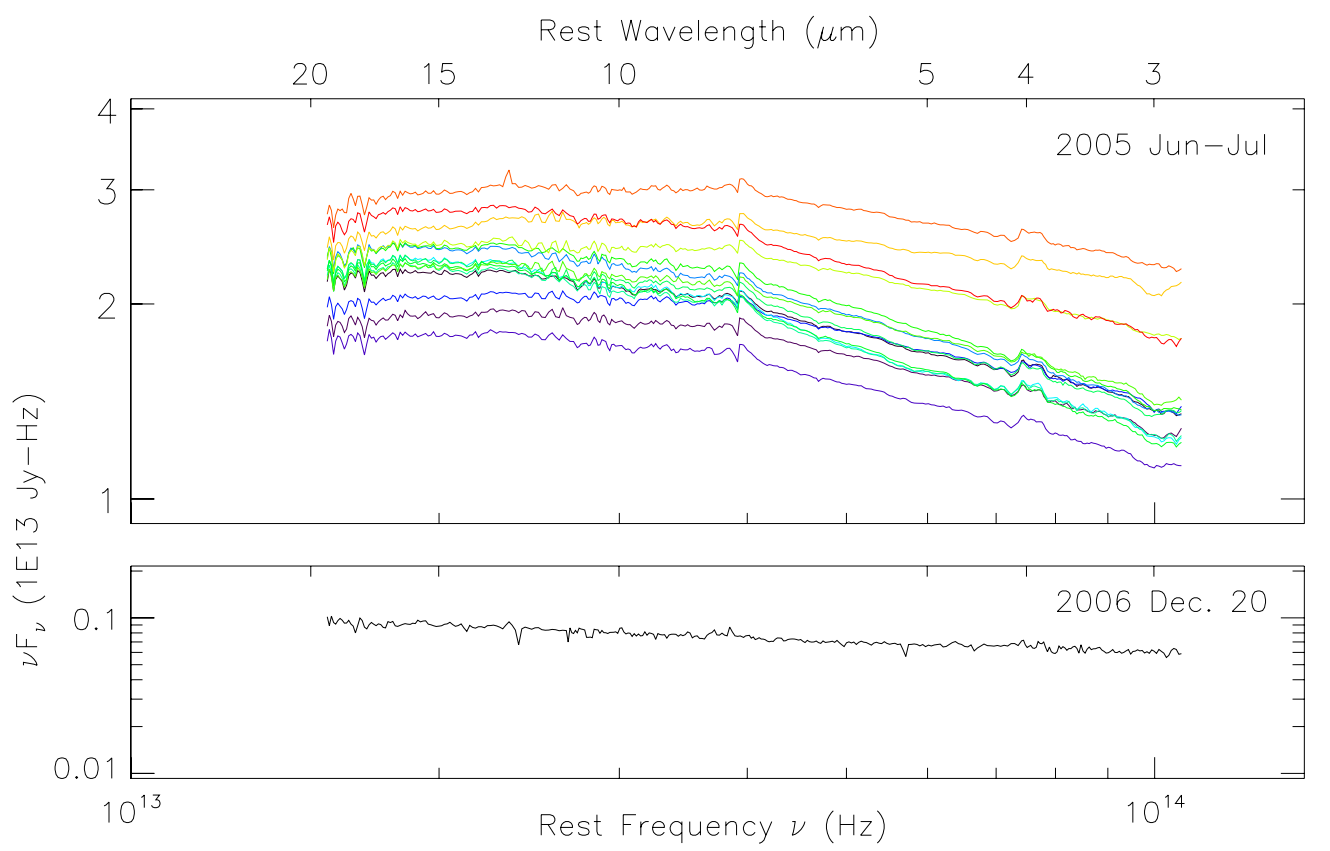

Figure 5. Spitzer IRS low-resolution spectra. Top: 2005 June-July. The bumps at $4 \times 10^{13} \mathrm{~Hz}$ and $7.3 \times 10^{13} \mathrm{~Hz}(14 \mu \mathrm{m}$ and $7 \mu \mathrm{m}$, observed $)$ and the dip at $1 \times 10^{14} \mathrm{~Hz}$ $\left(\sim 5.5 \mu \mathrm{m}\right.$, observed) are instrumental features. The noise spike at $2.2 \times 10^{13} \mathrm{~Hz}$ (top spectrum) is an artifact of saturation. Other small bumps and wiggles in the spectra are attributable to residual detector fringes. Bottom: 2006 December 20 low-state spectrum.

set (BCD) level data was performed to obtain useful fluxes by MIPS Instrument Team member K. Gordon (Gordon et al. 2005). For 24 and $70 \mu \mathrm{m}$ data, the pipeline mosaic data (version 13.2.0) were adequate for our use. The 2007 January 2 data were not affected by soft saturation; we used pipeline data (version 13.2.0). The IPAC Skyview analysis package was used for aperture photometry. At $24 \mu \mathrm{m}$, we used a 13" radius aperture and applied an aperture correction of 1.164 (Table 3.13, p. 33 of MIPS Data Handbook, version 3.2). At $70 \mu \mathrm{m}$, we used $35^{\prime \prime}$ radius aperture and applied an aperture correction for a $v^{-2}$ spectral index of 1.197 (Table 3.14, p. 33, MIPS Data Handbook, version 3.2). For our $160 \mu \mathrm{m}$ data on 2007 January 2 , we used $50^{\prime \prime}$ radius aperture and applied an aperture correction of 1.470 (Table 3.16, p. 35, MIPS Data Handbook, version 3.2).

The fluxes on 2005 July 27 at 01:48 UT and 15:11 UT were 1.12 and $1.16 \mathrm{Jy}$ at $24 \mu \mathrm{m}, 3.51 \mathrm{Jy}$ at $70 \mu \mathrm{m}$, and $5.43 \mathrm{Jy}$ at $160 \mu \mathrm{m}$, with systematic errors of $10 \%, 10 \%$, and $20 \%$, respectively. The fluxes on 2007 January 2 at 24,70 , and $160 \mu \mathrm{m}$ were $0.060 \mathrm{Jy}, 0.189 \mathrm{Jy}$, and $0.22 \mathrm{Jy}$, with systematic errors of $10 \%, 10 \%$, and $30 \%$, respectively.

Additional confidence in the Spitzer low-state $160 \mu \mathrm{m}$ flux density of $0.22 \mathrm{Jy}$ with $30 \%$ systematic uncertainty, measured on 2007 January 2 , is provided by the completely independent Infrared Space Observatory Photometer measurements while the quasar was in an earlier low state: ISO-Phot measured the 120 and $180 \mu \mathrm{m}$ flux densities of 0.187 and $0.248 \mathrm{Jy}$ on 1997 December 18 (Haas et al. 2004). ISO-PHOT and Spitzer MIPS were calibrated using different primary and secondary calibrators, including asteroids. The 3C454.3 maps made by ISO and Spitzer were in different orientations on the sky.

\subsubsection{IRS Low Resolution}

IRS was used in standard staring mode to take low-resolution spectra with the Short-Low (SL2,1) and Long-Low (LL2,1) modules (Figure 5). Because of the unexpectedly bright flux level, digital saturation occurred in some of the 2005 July exposure ramps. This effect is automatically corrected for in the data reduction pipeline by throwing out the saturated samples, leading to lower effective exposure time for the brightest pixels and a corresponding reduction in the signal-to-noise ratio $(\mathrm{S} / \mathrm{N})$. Several pixels in the LL1 order of the epoch 14 spectrum had only two unsaturated samples in each ramp, leading to the noise spike at $22 \mu \mathrm{m}$. To guard against this problem, we reduced the exposure times and increased the number of exposures in subsequent epochs.

Spectral reductions began from the BCDs, which have been processed using the Spitzer S15.3.0 pipeline. The pipeline applied subtraction of dark current, ramp fitting, and nonlinearity, droop, and stray-light corrections. The two-dimensional BCD spectra were median-averaged at each of the two standard nod positions and off-source sky background was subtracted. Radiation-damaged "rogue" pixels were interactively selected and removed from the two-dimensional spectra using the IRSCLEAN program. Spectra for each nod position were extracted using standard tapered windows (SL2: 7".2 at $6 \mu \mathrm{m}$, SL1: $144^{\prime \prime} 4$ at $12 \mu \mathrm{m}$, LL2: 20 '. 4 at $16 \mu \mathrm{m}$, LL1: $34 . " 4$ at $27 \mu \mathrm{m}$ ). Finally, spectra from the two nod positions were averaged.

We have devised a fringe-correction algorithm to ameliorate spectral fringes in the LL spectra. The wavelengths of the detector fringes depend on where exactly the source is placed in the slit, which varies with the pointing error of the telescope. The detector fringe patterns were extracted from flat-field images and used to derive fringe-correction functions. The fringe spectrum was shifted and divided by itself to generate a correction curve to minimize the residual fringes in the source spectrum. This procedure reduced the residual fringe amplitudes from $<4 \%$ to $<2 \%$ in LL.

We found significant offsets between low-resolution spectral orders, which were likely caused by random pointing offsets. This effect was most pronounced in the SL1 order because of the small size of the slit relative to the telescope PSF. Multiplicative corrections of 1.0-1.2 (constant with wavelength) were applied 
Table 5

Spitzer IRS mid-IR Spectrophotometry of 3C 454.3

\begin{tabular}{lccccccccccc}
\hline \hline Epoch & Day $^{\mathrm{a}}$ & $F_{6}{ }^{\mathrm{b}}$ & $+/-$ & $F_{12}{ }^{\mathrm{c}}$ & $+/-$ & $F_{18}{ }^{\mathrm{d}}$ & $+/-$ & $F_{24} \mathrm{e}^{\mathrm{d}}+/-$ & $F_{30}^{\mathrm{f}}$ & $+/-$ \\
\hline 1 & 0.0721 & 0.29 & 0.02 & 0.74 & 0.03 & 1.26 & 0.04 & 1.80 & 0.05 & 2.24 & 0.09 \\
2 & 1.2601 & 0.27 & 0.02 & 0.68 & 0.02 & 1.12 & 0.04 & 1.57 & 0.04 & 1.91 & 0.07 \\
3 & 2.2021 & 0.24 & 0.02 & 0.62 & 0.02 & 1.03 & 0.03 & 1.45 & 0.04 & 1.79 & 0.07 \\
4 & 2.9945 & 0.29 & 0.02 & 0.74 & 0.02 & 1.22 & 0.04 & 1.67 & 0.04 & 2.04 & 0.07 \\
5 & 4.0043 & 0.29 & 0.02 & 0.79 & 0.03 & 1.36 & 0.05 & 1.95 & 0.06 & 2.45 & 0.10 \\
6 & 5.4844 & 0.27 & 0.02 & 0.72 & 0.03 & 1.27 & 0.04 & 1.86 & 0.06 & 2.34 & 0.09 \\
7 & 6.3560 & 0.26 & 0.02 & 0.71 & 0.03 & 1.25 & 0.04 & 1.81 & 0.06 & 2.28 & 0.09 \\
8 & 7.1905 & 0.28 & 0.02 & 0.76 & 0.03 & 1.30 & 0.05 & 1.86 & 0.06 & 2.34 & 0.09 \\
9 & 8.1915 & 0.26 & 0.02 & 0.72 & 0.03 & 1.27 & 0.05 & 1.83 & 0.05 & 2.31 & 0.09 \\
10 & 9.0716 & 0.29 & 0.02 & 0.82 & 0.03 & 1.40 & 0.05 & 1.98 & 0.06 & 2.45 & 0.09 \\
11 & 10.1821 & 0.30 & 0.02 & 0.79 & 0.03 & 1.32 & 0.05 & 1.85 & 0.05 & 2.28 & 0.08 \\
12 & 11.2788 & 0.37 & 0.03 & 0.91 & 0.03 & 1.46 & 0.04 & 2.01 & 0.06 & 2.47 & 0.09 \\
13 & 11.9932 & 0.43 & 0.03 & 1.02 & 0.03 & 1.60 & 0.05 & 2.18 & 0.05 & 2.60 & 0.09 \\
14 & 12.9880 & 0.47 & 0.03 & 1.14 & 0.03 & 1.80 & 0.05 & 2.46 & 0.07 & 2.95 & 0.10 \\
15 & 13.9533 & 0.37 & 0.03 & 0.95 & 0.03 & 1.61 & 0.06 & 2.27 & 0.06 & 2.79 & 0.10 \\
16 & 538.5000 & 0.012 & 0.001 & 0.028 & 0.001 & 0.047 & 0.002 & 0.070 & 0.003 & 0.092 & 0.004 \\
\hline
\end{tabular}

Notes.

a Start time of exposure (days) from UT 2005 June 30 00:00 (MJD 53551).

${ }^{\mathrm{b}}$ Mean 5.5-6.5 $\mu \mathrm{m}(3.0-3.5 \mu \mathrm{m}$ rest) flux density (Jy).

${ }^{c}$ Mean 11.5-12.5 $\mu \mathrm{m}(6.2-6.7 \mu \mathrm{m}$ rest) flux density (Jy).

${ }^{\mathrm{d}}$ Mean 17-19 $\mu \mathrm{m}$ (9.1-10.2 $\mu \mathrm{m}$ rest) flux density (Jy).

e Mean 23-25 $\mu \mathrm{m}$ (12.4-13.4 $\mu \mathrm{m}$ rest) flux density (Jy).

f Mean 28-32 $\mu \mathrm{m}$ (15.1-17.2 $\mu \mathrm{m}$ rest) flux density (Jy).

to match the flux in each of the SL2, SL1, and LL2 spectral orders to LL1 to within an accuracy of $1 \%$. The corrections were largest in the epochs 6-8 SL1 spectra (8\%-20\%). For these epochs, there was a residual convex downward curvature of $\sim 3 \%$ in SL1 caused by wavelength dependent slit losses, which we did not correct, but which do not affect our conclusions.

We used flux calibrations provided by the SSC, employing low-order polynomial fits to standard star observations to convert from electron $\mathrm{s}^{-1}$ to $\mathrm{Jy}$. The absolute flux calibration accuracy is limited by the uncertainty of the stellar atmosphere model for the flux calibration standard HR 7341, which is estimated to be $3 \%-5 \%$ across the IRS wavelength band (Decin $\&$ Eriksson 2007; Decin et al. 2004). The relative flux accuracy of each epoch is limited by slit losses. Repeatability observations of the standard star HD 173511 give independent $1 \sigma$ flux calibration uncertainties of $2 \%$ for SL2, $1 \%$ for SL1 and LL2, and $3 \%$ for LL1. After matching all orders to LL1, the relative flux accuracy is 3\%. We achieve $\mathrm{S} / \mathrm{N}$ values of $\sim 60-120$ in the low-resolution spectra, limited by remaining systematic uncertainties in the flat field at a level of $1 \%-2 \%$.

We measure the mean flux in five wave bands (Table 5: $5.5-6.5,11.5-12.5,17-19,23-25$, and $28-32 \mu \mathrm{m}$, observed) from the IRS low-resolution spectra. The mean wavelengths for these bands are centered at 6.0, 12.0, 18.0, 24.0, and $30.0 \mu \mathrm{m}$. The light curves for these wave bands are presented in Section 4.2.

There are no emission lines or absorption lines stronger than $2 \%$ of the continuum flux in the 2005 June-July low-resolution spectra (Figure 5). There is also no evidence for any significant broad silicate absorption or emission or any emission from polycyclic aromatic hydrocarbons (PAHs). Small deviations of $<2 \%$ come from uncertainties in the flux calibration introduced by residual detector fringing effects. The most prominent instrumental feature is a $\sim 10 \%$ bump at $14 \mu \mathrm{m}$ (the so-called SL1 "teardrop"), which may be caused by scattered light in the detector. There is a similar instrumental bump at $7 \mu \mathrm{m}$ and a dip at $\sim 5.5 \mu \mathrm{m}$, both in SL2 and of unknown origin.

\subsubsection{Comparison of 2005 June-July with 2006 December IRS Observations}

The 2005 June-July Spitzer spectra (Figure 5) were taken more than 2 months after the peak of the 2005 optical outburst. Even so late after the peak, 3C 454.3 was a factor of $\sim 20$ brighter at mid-IR wavelengths than at any previously published epoch. The peak flux during this period reached $1.14 \pm 0.03 \mathrm{Jy}$ at $12 \mu \mathrm{m}$ and $2.46 \pm 0.07 \mathrm{Jy}$ at $24 \mu \mathrm{m}$ (Table 5). The mean spectrum was fit by a power law $F_{v} \sim v^{-1.2}$ at $6-12 \mu \mathrm{m}$, with an exponential cutoff at longer wavelengths. The primary synchrotron bump peaked at an observed frequency of (1-4) $\times 10^{13} \mathrm{~Hz}(30-7 \mu \mathrm{m})$.

In 2006 December, we found 3C 454.3 in a relatively low state (Figure 5). The low-state spectrum is well fit by a simple power law with spectral index $\alpha=1.3$. We find no evidence of emission lines or silicate emission stronger than $3 \%$ of the continuum. This suggests that the low-state IR SED is dominated by synchrotron emission from the jet. The flux was $28 \pm 1 \mathrm{mJy}$ at $12 \mu \mathrm{m}$, a factor of 40 down from the maximum in 2005 July and slightly fainter than the historical ISO measurement of $34 \mathrm{mJy}$ at $12.8 \mu \mathrm{m}$ (Haas et al. 2004).

\subsubsection{IRS High Resolution}

We also took high-resolution spectra (Figure 6) with the Short-High (SH) and Long-High (LH) modules at the beginning and end of the 2005 IRS campaign (June 30 and July 13). These observations were designed to search for any narrow emission or absorption features in the mid-IR spectrum. The high-resolution spectra are divided by a factor of 1.04 to match the lowresolution flux calibration, since there is a level mismatch between the two flux standards used in the S15 pipeline to calibrate low- and high-resolution data. ${ }^{6}$ No sky subtraction

\footnotetext{
6 http://ssc.spitzer.caltech.edu/irs/irsinstrumenthandbook/
} 

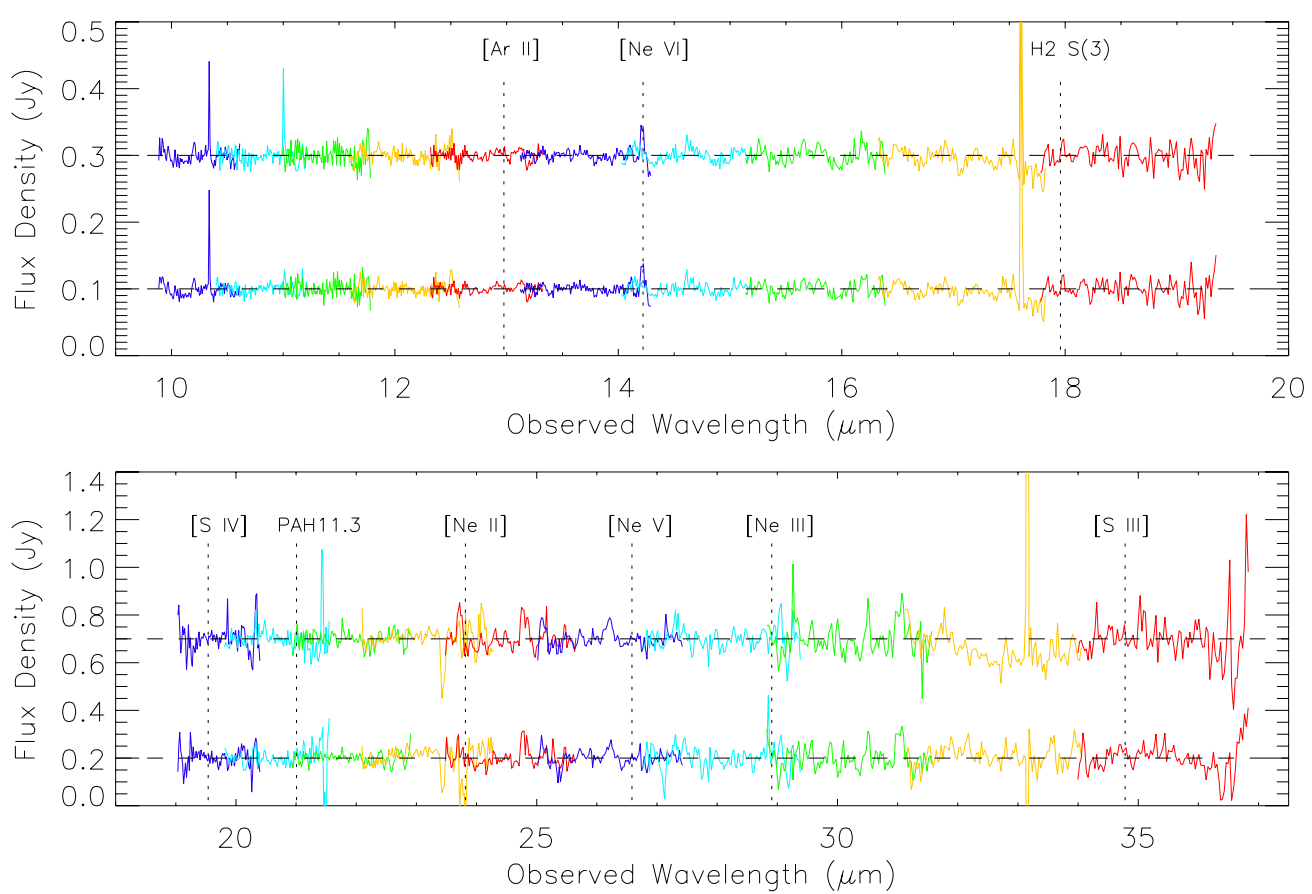

Figure 6. Continuum-subtracted high-resolution spectra of 3C 454.3. The 2005 June 30 and July 13 epochs are both offset from zero by an arbitrary amount for clarity. Top panel: SH; bottom panel: LH. No significant emission lines are detected. We do not consider the noise spike at the [Ne vI] line to be a detection because it is not seen in the adjacent order.

Table 6

Foggy Bottom Observatory $V$-band Observations of 3C 454.3

\begin{tabular}{lcccccccc}
\hline \hline Year & Month & Day & UT & Julian Date & $V$ mag & $V$ mag Error & $\begin{array}{c}\text { Flux } \\
(\mathrm{mJy})\end{array}$ & $\begin{array}{c}\text { Flux Error } \\
(\mathrm{mJy})\end{array}$ \\
\hline 2005 & 5 & 9 & $8: 42$ & 2453499.863 & 12.33 & 0.031 & 36.01 & 1.112 \\
2005 & 5 & 10 & $8: 39$ & 2453500.861 & 12.804 & 0.031 & 23.269 & 0.713 \\
2005 & 5 & 13 & $8: 31$ & 2453503.855 & 13.264 & 0.032 & 15.242 & 0.484 \\
2005 & 5 & 17 & $8: 19$ & 2453507.847 & 13.585 & 0.035 & 11.337 & 0.397 \\
2005 & 5 & 19 & $7: 57$ & 2453509.832 & 12.697 & 0.029 & 25.697 & 0.734 \\
2005 & 5 & 21 & $8: 02$ & 2453511.835 & 13.509 & 0.034 & 12.159 & 0.414 \\
2005 & 5 & 26 & $7: 43$ & 2453516.822 & 13.657 & 0.027 & 10.606 & 0.29 \\
2005 & 5 & 28 & $8: 09$ & 2453518.84 & 13.868 & 0.047 & 8.741 & 0.412 \\
2005 & 5 & 30 & $7: 52$ & 2453520.828 & 13.96 & 0.045 & 8.026 & 0.358 \\
2005 & 5 & 31 & $7: 43$ & 2453521.822 & 14.228 & 0.063 & 6.269 & 0.394 \\
\hline
\end{tabular}

(This table is available in its entirety in a machine-readable form in the online journal. A portion is shown here for guidance regarding its form and content.)

was performed. The sky background was small compared to the source flux in SH for the brighter of the two epochs. However, it became significant for the larger LH slit and for the fainter epoch.

We subtracted a second-order polynomial fit to each order of the high-resolution spectra in order to get a closer look at any possible emission line features (Figure 6). On inspection, it is clear that there is no significant detection at either epoch of any of the following quasar or host galaxy emission features at a redshift of $z=0.859$ : [Ar II] $6.99 \mu \mathrm{m}, \mathrm{H}_{2} \mathrm{~S}(3) 9.66 \mu \mathrm{m}$, [S IV] $10.51 \mu \mathrm{m}$, PAH $11.3 \mu \mathrm{m}$, [Ne II] $12.81 \mu \mathrm{m}$, [Ne v] $14.3 \mu \mathrm{m}$, [Ne III] $15.56 \mu \mathrm{m}$, or [S III] $18.71 \mu \mathrm{m}$. The noise feature at [Ne VI] $\lambda 7.65 \mu \mathrm{m}$ (observed $14.22 \mu \mathrm{m}$ ) must be spurious since it is not duplicated in the adjacent order. It is most likely caused by an instrumental defect or noise at the edge of SH order 14.

\subsection{Optical Observations}

Since 1988, T. Balonek has conducted blazar monitoring observations with Colgate University's Foggy Bottom Observa- tory. As described by Kartaltepe \& Balonek (2007), observations are made "using a Ferson 16 inch Newtonian/Cassegrain reflecting telescope equipped with a Star I CCD system. The images range in exposure time from 1 to 5 minutes (most images being 2 minutes) and include the $B, V, R$, and $I$ filters, designed by Beckert (1989) to conform to the Johnson-Cousins system. . . The images were reduced using standard IRAF packages and customized scripts written to facilitate the data handling for our system." In the case of 3C 454.3, $V$-, $R$-, and I-band data were obtained. The photometry for all of the images was calculated using the IRAF apphot package with a 16" diameter aperture and a sky annulus with an inner diameter of $20^{\prime \prime}$ and an outer diameter of $40^{\prime \prime}$. Some of the Colgate data were previously shown by Raiteri et al. (2008b). The Foggy Bottom Observatory data, uncorrected for absorption, are listed in Tables 6-8.

Since 2005, the members of the NASA Space Interferometry Mission Key Project on Active Galactic Nuclei, led by A. Wehrle, have conducted blazar monitoring observations using the Caltech automated $1.5 \mathrm{~m}$ telescope at Palomar Observatory 
Table 7

Foggy Bottom Observatory $R$-band Observations of 3C 454.3

\begin{tabular}{lcrrlcccc}
\hline \hline Year & Month & Day & UT & Julian Date & $R$ mag & $R$ mag Error & $\begin{array}{c}\text { Flux } \\
(\mathrm{mJy})\end{array}$ & $\begin{array}{c}\text { Flux Error } \\
(\mathrm{mJy})\end{array}$ \\
\hline 2004 & 6 & 21 & $7: 41$ & 2453177.82 & 15.315 & 0.019 & 2.304 & 0.044 \\
2004 & 7 & 7 & $7: 25$ & 2453193.809 & 15.189 & 0.048 & 2.588 & 0.124 \\
2004 & 7 & 21 & $7: 21$ & 2453207.806 & 15.268 & 0.052 & 2.406 & 0.126 \\
2004 & 8 & 22 & $4: 45$ & 2453239.698 & 15.66 & 0.042 & 1.677 & 0.071 \\
2004 & 8 & 25 & $7: 00$ & 2453242.792 & 15.92 & 0.134 & 1.32 & 0.176 \\
2004 & 9 & 5 & $3: 27$ & 2453253.644 & 14.803 & 0.029 & 3.692 & 0.108 \\
2004 & 9 & 12 & $7: 05$ & 2453260.796 & 15.212 & 0.045 & 2.534 & 0.113 \\
2004 & 9 & 14 & $4: 40$ & 2453262.695 & 14.419 & 0.017 & 5.261 & 0.087 \\
2004 & 9 & 19 & $4: 37$ & 2453267.692 & 14.356 & 0.015 & 5.573 & 0.082 \\
2005 & 5 & 9 & $8: 33$ & 2453499.857 & 11.898 & 0.007 & 53.619 & 0.398 \\
\hline
\end{tabular}

(This table is available in its entirety in a machine-readable form in the online journal. A portion is shown here for guidance regarding its form and content.)

Table 8

Foggy Bottom Observatory I-band Observations of 3C 454.3

\begin{tabular}{lcrrlllll}
\hline \hline Year & Month & Day & UT & Julian Date & $I$ mag & $I$ mag Error & $\begin{array}{c}\text { Flux } \\
(\mathrm{mJy})\end{array}$ & $\begin{array}{c}\text { Flux Error } \\
(\mathrm{mJy})\end{array}$ \\
\hline 2005 & 5 & 9 & $8: 49$ & 2453499.868 & 11.22 & 0.018 & 100.12 & 1.828 \\
2005 & 5 & 10 & $8: 47$ & 2453500.867 & 11.541 & 0.019 & 74.467 & 1.412 \\
2005 & 5 & 13 & $8: 42$ & 2453503.863 & 12.157 & 0.017 & 42.259 & 0.715 \\
2005 & 5 & 17 & $8: 30$ & 2453507.854 & 12.367 & 0.012 & 34.822 & 0.432 \\
2005 & 5 & 19 & $7: 40$ & 2453509.82 & 11.522 & 0.009 & 75.806 & 0.664 \\
2005 & 5 & 19 & $8: 26$ & 2453509.852 & 11.529 & 0.01 & 75.317 & 0.775 \\
2005 & 5 & 21 & $8: 17$ & 2453511.846 & 12.309 & 0.01 & 36.709 & 0.383 \\
2005 & 5 & 26 & $7: 57$ & 2453516.832 & 12.445 & 0.01 & 32.399 & 0.322 \\
2005 & 5 & 28 & $8: 22$ & 2453518.849 & 12.659 & 0.014 & 26.596 & 0.379 \\
2005 & 5 & 30 & $8: 03$ & 2453520.836 & 12.888 & 0.014 & 21.545 & 0.297 \\
\hline
\end{tabular}

(This table is available in its entirety in a machine-readable form in the online journal. A portion is shown here for guidance regarding its form and content.)

Table 9

Palomar Observatory $V$-band Observations of 3C 454.3

\begin{tabular}{lcccccccc}
\hline \hline Year & Month & Day & UT & Julian Date & $V$ mag & $V$ mag Error & $\begin{array}{c}\text { Flux } \\
(\mathrm{mJy})\end{array}$ & $\begin{array}{c}\text { Flux Error } \\
(\mathrm{mJy})\end{array}$ \\
\hline 2005 & 05 & 12 & $11: 40$ & 2453502.9861 & 13.271 & 0.002 & 17.894 & 0.033 \\
2005 & 05 & 13 & $11: 40$ & 2453503.9861 & 13.410 & 0.002 & 15.744 & 0.029 \\
2005 & 05 & 16 & $10: 54$ & 2453506.9542 & 13.414 & 0.002 & 15.686 & 0.029 \\
2005 & 05 & 18 & $11: 37$ & 2453508.9840 & 13.074 & 0.002 & 21.454 & 0.040 \\
2005 & 05 & 20 & $11: 11$ & 2453510.9660 & 13.340 & 0.002 & 16.792 & 0.031 \\
2005 & 05 & 23 & $10: 34$ & 2453513.9403 & 13.854 & 0.004 & 10.459 & 0.039 \\
2005 & 05 & 24 & $11: 10$ & 2453514.9653 & 13.521 & 0.003 & 14.214 & 0.039 \\
2005 & 05 & 30 & $10: 06$ & 2453520.9208 & 14.139 & 0.004 & 8.045 & 0.030 \\
2005 & 05 & 31 & $10: 19$ & 2453521.9299 & 14.345 & 0.004 & 6.654 & 0.025 \\
2005 & 06 & 04 & $09: 18$ & 2453525.8875 & 14.162 & 0.003 & 7.876 & 0.022 \\
\hline
\end{tabular}

(This table is available in its entirety in a machine-readable form in the online journal. A portion is shown here for guidance regarding its form and content.)

(Cenko et al. 2006). We intensified monitoring of 3C 454.3 when it flared in 2005. Data in filter bands $V$ and $R$ were obtained. ${ }^{7}$ Data were reduced with IRAF aperture photometry within a 2 '. 28 radius, using a sky annulus of 3 ". 8 radius and 3 ". 8 width. The comparison star sequence is given online ${ }^{8}$ and originally

7 Note that the redshift of $3 \mathrm{C} 454.3$ brings the broad emission lines from C III] (1909 $\AA$ rest, $3549 \AA$ observed) into the blue edge of the $B$-band filter and $\mathrm{Mg}$ II (2798 $\AA$ rest, $5201 \AA$ observed) into both $B$ and $V$ filters. See Pian et al. (2005) for Hubble Faint Object Spectrograph spectra of 3C 454.3 taken in 1991 and 1995.

8 http://www.lsw.uni-heidelberg.de/projects/extragalactic/charts/ by Craine (1977), Angione (1971), Fiorucci et al. (1998), Smith \& Balonek (1998), and Raiteri et al. (1998). We used Star H, with $V=13.64$ and $R=13.10$, as the primary comparison star. The Palomar Observatory data, uncorrected for absorption, are given in Tables 9 and 10.

\subsection{Chandra Observations}

Chandra HETG observations were taken nearly simultaneously with our Spitzer MIPS observations, for $2.145 \mathrm{ks}$ from 21:35 UT to 22:42 UT on 2007 January 1. The grating 
Table 10

Palomar Observatory $R$-band Observations of $3 C 454.3$

\begin{tabular}{lcccccccc}
\hline \hline Year & Month & Day & UT & Julian Date & $R$ mag & $R$ mag Error & $\begin{array}{c}\text { Flux } \\
(\mathrm{mJy})\end{array}$ & $\begin{array}{c}\text { Flux Error } \\
(\mathrm{mJy})\end{array}$ \\
\hline 2005 & 5 & 12 & $11: 40$ & 2453502.9861 & 12.721 & 0.002 & 25.128 & 0.046 \\
2005 & 5 & 13 & $11: 40$ & 2453503.9861 & 12.860 & 0.002 & 22.108 & 0.041 \\
2005 & 5 & 16 & $10: 54$ & 2453506.9542 & 12.864 & 0.002 & 22.027 & 0.041 \\
2005 & 5 & 18 & $11: 37$ & 2453508.9840 & 12.524 & 0.002 & 30.127 & 0.056 \\
2005 & 5 & 20 & $11: 11$ & 2453510.9660 & 12.790 & 0.002 & 23.580 & 0.043 \\
2005 & 5 & 23 & $10: 34$ & 2453513.9403 & 13.304 & 0.004 & 14.688 & 0.054 \\
2005 & 5 & 24 & $11: 10$ & 2453514.9653 & 12.971 & 0.003 & 19.960 & 0.055 \\
2005 & 5 & 30 & $10: 06$ & 2453520.9208 & 13.589 & 0.004 & 11.297 & 0.042 \\
2005 & 5 & 31 & $10: 19$ & 2453521.9299 & 13.795 & 0.004 & 9.344 & 0.034 \\
2005 & 6 & 4 & $9: 18$ & 2453525.8875 & 13.612 & 0.003 & 11.060 & 0.031 \\
\hline
\end{tabular}

(This table is available in its entirety in a machine-readable form in the online journal. A portion is shown here for guidance regarding its form and content.)

was used to reduce potential photon pile up effects on the ACIS-S detector. The data were reduced using standard point-source grating analysis threads under CIAO 4.0. The Medium Energy Grating and High Energy Grating \pm 1 order grating spectra were grouped to have a minimum of 20 counts bin $^{-1}$. We fixed the hydrogen column density for model fits at $N_{\mathrm{H}}=6.5 \times 10^{20} \mathrm{~cm}^{-2}$ (Dickey \& Lockman 1990) and assumed standard elemental abundances to estimate Galactic absorption. The best fit to the $\mathrm{X}$-ray continuum was obtained with a single power law of slope $\Gamma=1.53 \pm 0.12$ and flux at $2-10 \mathrm{keV}$ of $(5.8 \pm 0.2) \times 10^{-12} \mathrm{erg} \mathrm{cm}^{-2} \mathrm{~s}^{-1}\left(8.14 \times 10^{-4}\right.$ photons $\mathrm{s}^{-1} \mathrm{~cm}^{-2}$ ). Adding absorption intrinsic to the host galaxy to the model did not improve the fit. Similar fits were obtained using either $\chi^{2}$ or C-statistics. We report fit parameters using the latter, because of small photon number statistics in spite of grouping the data.

\section{RESULTS}

\subsection{Optical Light Curves}

The optical light curves are shown in Figure 7. We scaled the Colgate $V$-band data by 1.15 to match the Palomar $V$ data in constructing the light curves; the discrepancy is probably due to slight mismatches in filters. The optical light curves give the appearance of a series of flares superimposed on an exponential fading of the big flare in 2005 May, with $e$-folding time of $\sim 60$ days. The source's underlying $V$-band flux faded from $23 \mathrm{mJy}$ on about 2005 May 10 to $1.1 \mathrm{mJy}$ on about 2005 September 9. Superimposed flares, numbering about a dozen between May and September, doubled the flux on timescales of 24-48 hr, with similar fade times.

\subsection{Infrared Light Curves}

The 2005 June-July infrared light curves for IRS and IRAC are shown together with the optical light curves in Figure 7. The IRS portions of these curves are shown in more detail in Figures 8(a) and (b). We compute mean 6-12, 6-24, and 12-24 $\mu \mathrm{m}$ spectral indices to characterize the spectral hardness, seen in Figure 8(c). The light curves have similar shapes in all mid-IR wave bands, with greatest amplitude at the shortest wavelengths. The $6 \mu \mathrm{m}$ flux increases by a factor of two during 2005 July from $240 \pm 20 \mathrm{mJy}$ at epoch 3 , flaring to $470 \pm$ $30 \mathrm{mJy}$ at epoch 14 . All of the dips and peaks in the light curves occur simultaneously at 6,12 , and $24 \mu \mathrm{m}$, to within 0.5 day. There is no measurable lag.

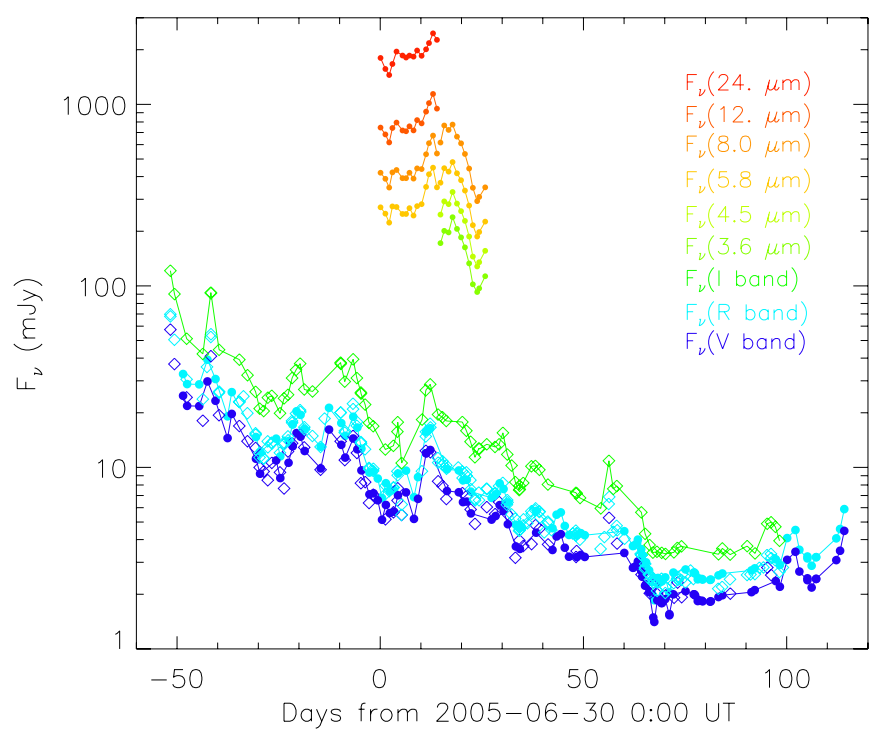

Figure 7. Optical and mid-IR light curves for 2005 May to October. Optical $V, R$, and $I$ band data from Foggy Bottom Observatory and Palomar Observatory are corrected for Galactic extinction and reddening. Mid-IR data are from the 2005 June 30 to July 26 Spitzer IRS and IRAC campaigns. The optical fluxes showed flaring behavior superposed on an overall decline. Measurement uncertainties are generally smaller than the plot symbols.

The variability, characterized by the fractional excess variance (Vaughan et al. 2003, Equation (10)) in Figure 8(d), decreases from $20 \% \pm 2 \%$ at $6 \mu \mathrm{m}$ to $12 \% \pm 1 \%$ at $30 \mu \mathrm{m}$. The variability amplitude may be moderated by optical depth to synchrotron self-absorption, which increases with wavelength. In principle, dilution by non-variable thermal dust emission from the AGN or host galaxy could also reduce the variability amplitude at longer wavelengths. The IRS low-state spectrum (Figure 5) strictly limits the thermal dust contribution to $<5 \%$ of the high-state spectrum. This is marginally insufficient to provide the $8 \% \pm 2 \%$ dilution that would be required to account for the decreased variability at $30 \mu \mathrm{m}$. Furthermore, the lack of dust emission features in the low-state IR spectrum (Section 3.2) and the shape of the low-state IR SED together with its hyperluminous infrared galaxy (LIRG) IR luminosity (Section 5.1) make such a scenario even more unlikely.

The 6-24 $\mu \mathrm{m}$ spectral index varies from a maximum of $1.41 \pm 0.06$ at epoch 9 to a minimum of $1.17 \pm 0.06$ at epoch 13 (Figure 8(c)). The spectral index uncertainties correspond to 

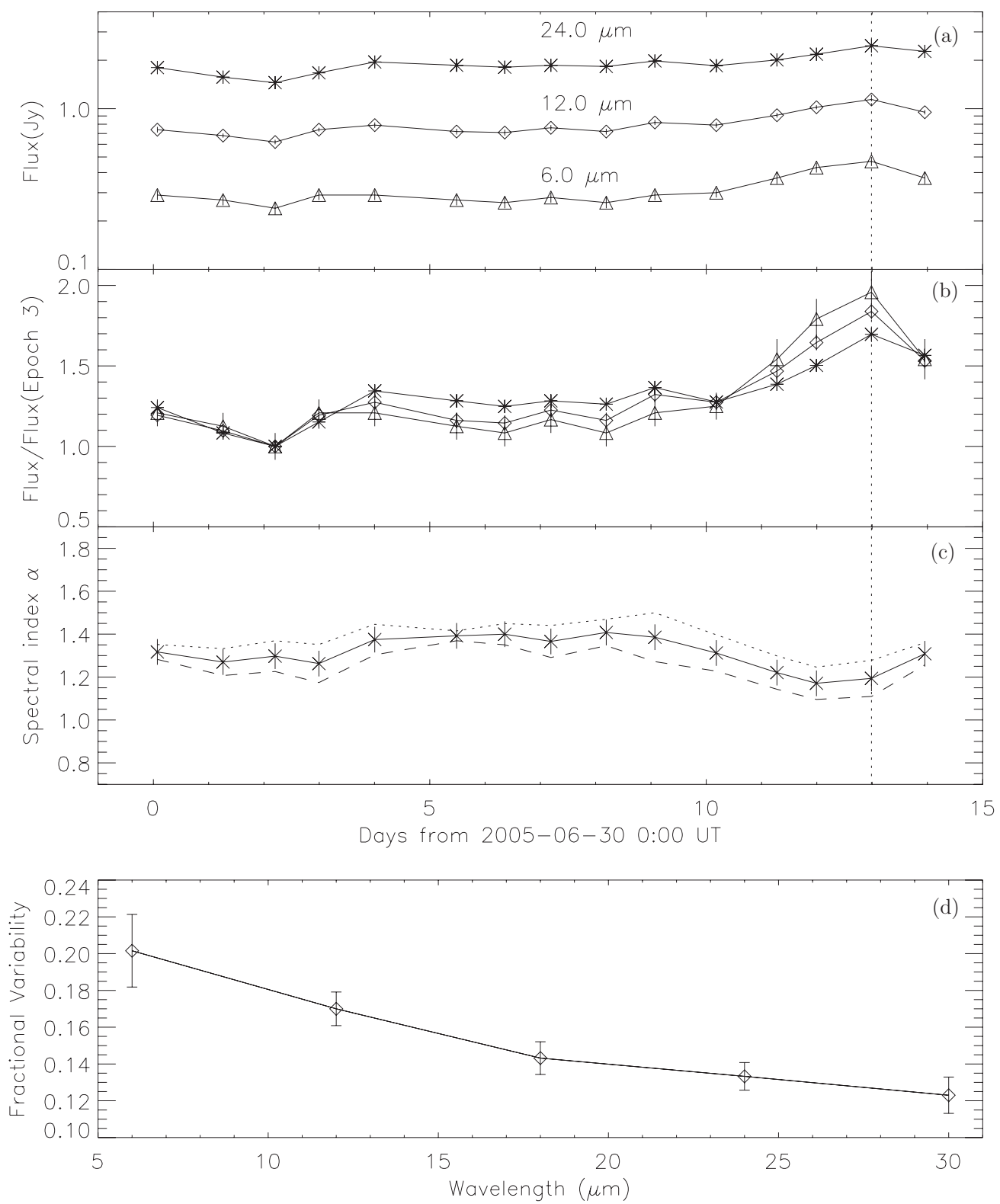

Figure 8. Spitzer IRS mid-IR variability in selected bands for 2005 June 30 to July 14. (a) Logarithmic scale; (b) normalized in each band to the flux at epoch 3 . (c) Spectral index curves, solid line $=6-24 \mu \mathrm{m}$ index, dotted line $=6-12 \mu \mathrm{m}$ index, and dashed line $=12-24 \mu \mathrm{m}$ index. The spectrum becomes harder during the bright flare that peaks on 2005 July 13, indicated with a vertical line. (d) The variability (fractional excess variance) decreases with wavelength in the IRS bands.

the IRS flux repeatability of $2 \%$ at $6 \mu \mathrm{m}$ and $3 \%$ at $24 \mu \mathrm{m}$, as given in Section 3.2.3. As the flux increases from epochs 4-5, the slope becomes softer. The flux and spectral slope show only small changes from epochs $5-10$. This period is followed by a large flare from epochs $11-14$, when the flux increases by $85 \%$ at $6 \mu \mathrm{m}$ and the spectrum hardens. The flux falls and the spectrum softens again in the epoch after the peak of the flare. This flare behavior is characterized by an anticorrelation between spectral index and $6 \mu \mathrm{m}$ flux (Figure 9). The SED becomes harder at high frequencies as the flux increases and the SED peak also moves to higher frequency: from $(1.8-2.4) \times 10^{13} \mathrm{~Hz}$ to $(2.2-4.0) \times 10^{13} \mathrm{~Hz}$.

\subsection{Correlation of Optical and Infrared Light Curves}

An inspection of the optical through infrared light curves (Figure 7) reveals correlated variability from the $V$ band to $24 \mu \mathrm{m}$. In particular the epoch 14 flare seen in the IRS light curves (Figure 8) is seen in all of the optical bands as well. However, a close comparison of the IRAC/IRS $8.0 \mu \mathrm{m}$ band with the $R$-band light curve (Figure 10) shows significant differences in flaring activity. ${ }^{9}$ In particular, the $8.0 \mu \mathrm{m}$ band shows a bright flare at days 15-20, following the epoch 14 flare, which is much less pronounced in the $R$ band. There are clear differences in the relative amplitudes of several other smaller flares, which change continuously with wavelength between the IR and optical bands. This behavior may indicate multiple flaring components in the 3C 454.3 SED which have peak amplitudes at different wavelengths. (See Section 4.4.)

A cross-correlation of the IRAC/IRS $8.0 \mu \mathrm{m}$ and $R$-band light curves shows a pronounced peak with correlation amplitude 0.7 at a lag of $0 \pm 0.5$ days (Figure 10(d)). This confirms the

\footnotetext{
9 We focus here on the joint IRAC/IRS $8.0 \mu \mathrm{m}$ band because it has the largest span (26 days) of simultaneous mid-IR coverage, and the $R$ band which has the best optical coverage.
} 


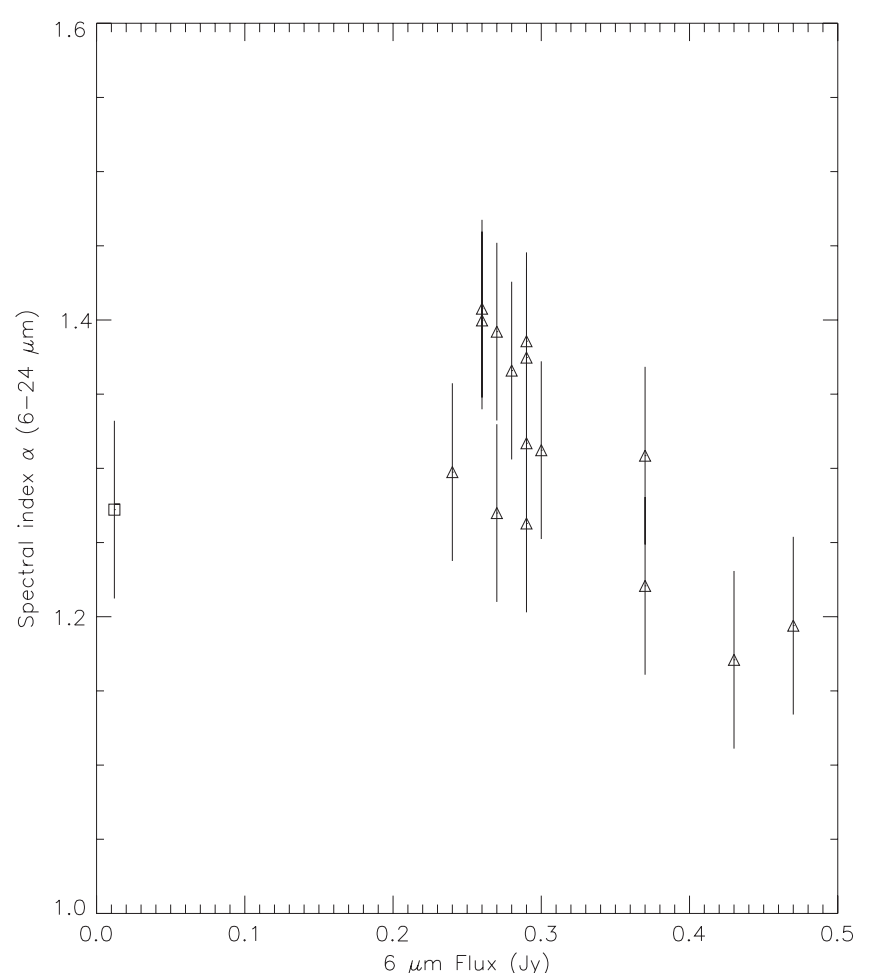

Figure 9. Color-flux diagram. The 6-24 $\mu \mathrm{m}$ spectral index is plotted vs. $6 \mu \mathrm{m}$ flux. Triangles: 2005 June-July, square: 2006 December. During the 2005 epochs, there is a general trend for harder spectral index at greater flux. However, there is not a 1:1 correspondence, likely because the flux and spectral index depend on the detailed history of each IR flare.

overall impression that the flaring activity is correlated across the optical and mid-IR bands. However, the cross-correlation curve is broadened, with a secondary peak at a lag of 4 days. This lag is similar to the time between the two brightest $8.0 \mu \mathrm{m}$ flares, which may produce an accidental (rather than causal) correlation signal.

\subsection{Mid-infrared-Optical SEDs}

The mid-infrared to optical SEDs are shown in Figure 11. From the figure, we observe the following.

1. During the period 2005 June 30 to July 13, there was less variation at IRS bands than in VRI bands, but during the following 10 days, 2005 July 14-25, there was more variation at IRAC bands than at VRI, including the 5.8 and $8.0 \mu \mathrm{m}$ bands that overlap in wavelength with the IRS.

2. The SED slope over mid-infrared through near-infrared to optical bands was negative in both bright (2005) and faint (2007) states. When the source was a factor of 6-10 weaker (in 2006 July and 2006 December) than it was during our 2005 July observations, Raiteri et al. (2007) observed that the underlying near-infrared-optical $(I-R-V$ bands) SED slope was positive, unlike our 2005 observations. In their data, the optical emission peaked at $V$, then decreased in Swift $B$ and $U$ bands and rose again at Swift $W 1$ and $M 2$ bands. These data, taken together, are consistent with the Raiteri et al. detection of an underlying "small blue bump" (probably a blend of iron lines, Mg II lines, and Balmer continuum from the BLR) whose $V$-band-peaked emission is visible only when the synchrotron emission is comparatively faint, and whose signature is overwhelmed by synchrotron emission when the source is flaring 6-10 times brighter. Our observations do not cover the
Swift bands which show the rise in thermal emission from the accretion disk.

3. The SED does not rise or fall by a constant amount across the mid-infrared to optical bands from day to day. Moreover, the individual band-to-band spectral indices are not the same from day to day. There is no obvious wavelength range over which we see the beginning and ending points of added emission. That implies the newly injected population of leptons giving rise to the synchrotron emission is broad in energy, but we have to keep in mind that the particles radiating at a given frequency are from both old and new populations. Previously injected populations contribute at lower frequencies long after that population's high-energy electrons no longer radiate at high frequencies, assuming no change in the ambient magnetic field.

4. Energy gained in a day at optical bands can be lost in a day, but it takes longer to both gain and lose energy at midinfrared bands. We see no evidence of asymmetric rise or fall in the mid-infrared or optical flares.

\subsection{Comparison of Outburst and Quiescent X-Ray Fluxes and Spectral Indices}

On 2005 May 19, during outburst and a month earlier than our first Spitzer observations, Chandra observed 3C 454.3 for 112 ks using the High Resolution Camera, Low Energy Transmission Grating, as a Target of Opportunity within a program on flaring blazars (PI: F. Nicastro; Villata et al. 2006). The $0.2-8 \mathrm{keV}$ spectrum was fitted with a power law of photon index $\Gamma=1.477 \pm 0.017$, with $N_{\mathrm{H}}=(1.34 \pm 0.05) \times$ $10^{21} \mathrm{~cm}^{-2}$, more than twice the Galactic value (a misprint in Villata et al. 2006 has been corrected). During our 2007 January 1 Chandra observations, obtained using the HETG mode when the source was quiescent, the $2-10 \mathrm{keV}$ spectral index was quite similar $(\Gamma=1.53 \pm 0.12)$ using the Galactic $N_{\mathrm{H}}$ value of $6.5 \times 10^{20} \mathrm{~cm}^{-2}$. The 2005 May de-absorbed fluxes were $F=(5.5 \pm 0.2) \times 10^{-11} \mathrm{erg} \mathrm{cm}^{-2} \mathrm{~s}^{-1}$ and $(8.4 \pm 0.2) \times 10^{-11}$ erg $\mathrm{cm}^{-2} \mathrm{~s}^{-1}$ in the $0.2-2 \mathrm{keV}$ and $2-8 \mathrm{keV}$ bands (respectively), compared to the 2007 January flux at $2-10 \mathrm{keV}$ of $(5.8 \pm 0.2) \times 10^{-12} \mathrm{erg} \mathrm{s}^{-1}$. Hence, the $2-10 \mathrm{keV} \mathrm{X}$-ray flux dropped by a factor of $\sim 10$ while the spectral index remained the same, but the bright state required twice as much $N_{\mathrm{H}}$ along the line of sight as the quiescent state. An INTEGRAL observation (Pian et al. 2006) made 2005 May 15-18 was fitted with a power law of index $\Gamma=1.8 \pm 0.1$ using the same (Dickey \& Lockman 1990) Galactic $N_{\mathrm{H}}$ value. The flux in the $3-200 \mathrm{keV}$ band was $5.45 \times 10^{-10} \mathrm{erg} \mathrm{cm}^{-2} \mathrm{~s}^{-1}$.

\section{DISCUSSION}

\subsection{The Spectral Energy Distributions: Two Synchrotron Peaks}

We use our multiwavelength photometry and the IRS spectra together in constructing radio-X-ray SEDs (Figure 12); representative data are listed for convenience in Tables 11 and 12. In constructing the SEDs, the photometry was corrected for Galactic absorption at $V, R$, and $I$ bands by $0.355,0.286$, and 0.208 mag, respectively. The Colgate $V$-band data were also scaled by 1.15 to match the Palomar $V$ data, as mentioned above.

During 2005 June 30 to July 12 (high state), the primary SED peak fell in the IRS 5-35 $\mu \mathrm{m}$ band (Figure 12). Strong variability of the continuum level, slope, and peak frequency are characteristic of synchrotron emission from a relativistic radio jet. The largest variability amplitudes are seen in the IRAC $3.6 \mu \mathrm{m}$ and near-IR $(I)$ bands and the smallest amplitude at 

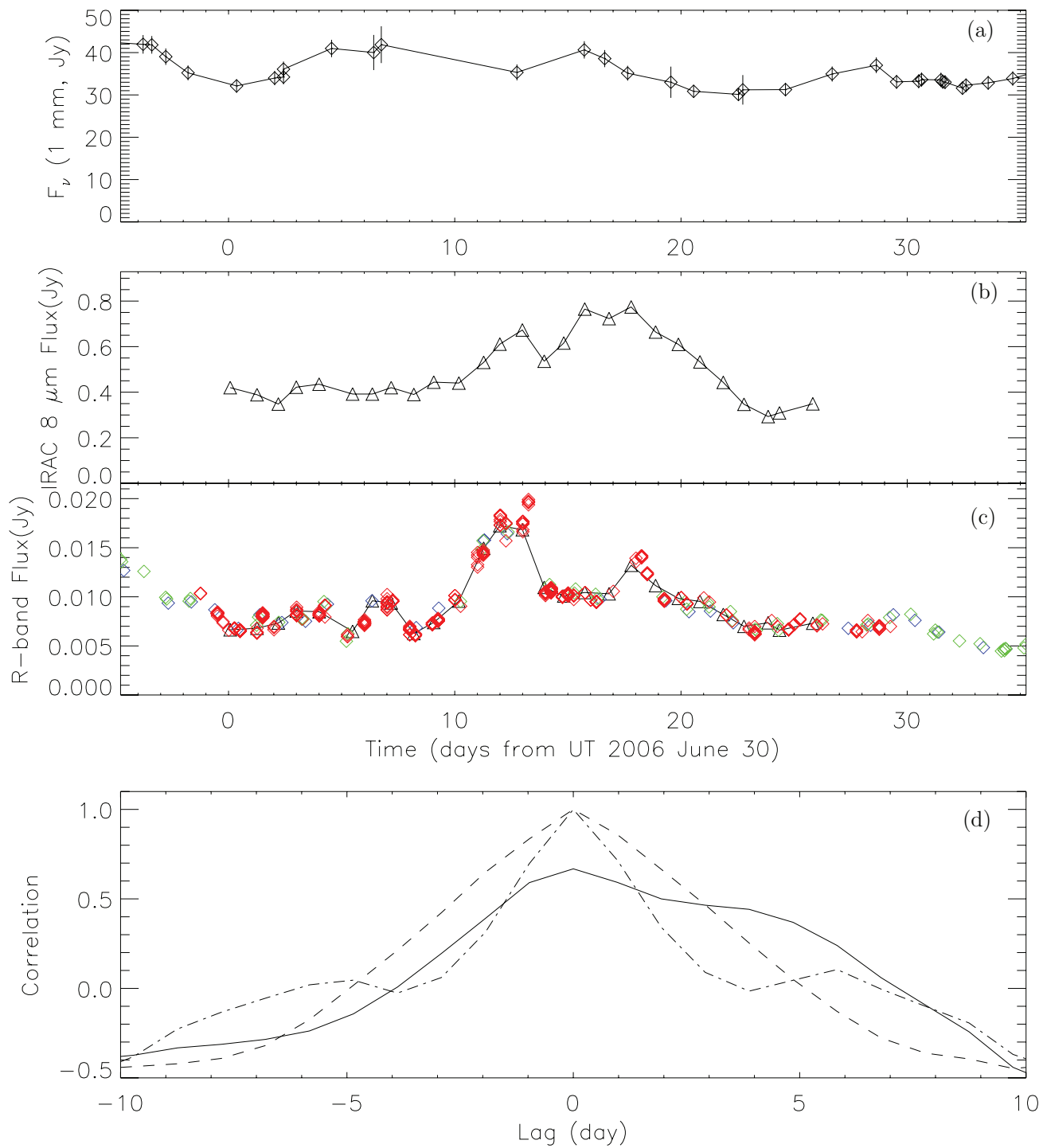

Figure 10. Comparison of (a) $1.3 \mathrm{~mm}(230 \mathrm{GHz}$ ) radio, (b) IRAC $8.0 \mu \mathrm{m}$, and (c) $R$-band light curves. WEBT $R$-band data (Villata et al. 2006) are included with the Colgate and Palomar data to improve the sampling during the 2005 June-July Spitzer observation period. The millimeter-wave variations do not appear to be correlated with the mid-IR variations, indicating that emission in the two bands comes from separate regions in the jet. (c) Cross-correlation of IRAC $8.0 \mu \mathrm{m}$ and $R$-band light curves (solid line). The autocorrelation curves for IRAC $8.0 \mu \mathrm{m}$ (dash), and $R$ band (dot-dashed) are shown for comparison. The IR and optical emission are strongly correlated at zero lag.

(A color version of this figure is available in the online journal.)

$35 \mu \mathrm{m}$. The turnover and lower variability amplitude at lower frequencies may indicate the onset of optically thick synchrotron emission. Without long-wavelength IR coverage, we lost track of the primary synchrotron peak location during the July 14-26 IRAC campaign. When the MIPS observations picked up on July 27 , the IR peak had faded somewhat and shifted to the MIPS $70 \mu \mathrm{m}$ band.

In the 2006 December 25 to 2007 January 1 low state, the IR SED peaked in the MIPS $70 \mu \mathrm{m}$ FIR band. The low-state SED was similar to that reported by Haas et al. (2004), and to other low-state measurements from NED published over the period 1979-1995 (Figure 12). One striking difference was the higher $60 \mu \mathrm{m}$ flux measured by Haas et al. (2004), which was $\sim 4$ times greater than our MIPS $70 \mu \mathrm{m}$ flux, but less than the 2005 peak. This is additional evidence that the $60 \mu \mathrm{m}$ flux is highly variable, and indicates that there was little if any contribution from thermal dust emission during the Haas et al. (2004) observation. It is particularly evident during our low-state Spitzer observations (Figure 12) that there are two peaks in the radio-IR SED, as seen earlier in the low-state observations, made by combining ISO data from 1996 December 12 and 1997 December 18, of Haas et al. (2004). As we recall from Section 3.2.2, the veracity of the Spitzer $160 \mu \mathrm{m}$ flux measurement in 2007 January is backed up by the detection of a similarly low level in 1997 by ISO.

During both the low and high states, the primary SED peak occurs at MIR-FIR wavelengths, depending on source brightness. The secondary peak occurs in the sub-mm, near $850 \mu \mathrm{m}$. Strong variability of both bumps indicates that they are both composed of synchrotron emission from the relativistic jet. At the time of the 2005 July 27 MIPS observations, two bumps are also apparent, with the minimum falling somewhere between $160 \mu \mathrm{m}$ and $850 \mu \mathrm{m}$. The sharpest contrast between the two peaks is seen during the ISO epochs, when the infrared peak was defined by the $0.706 \mathrm{Jy}$ peak at $60 \mu \mathrm{m}$. 


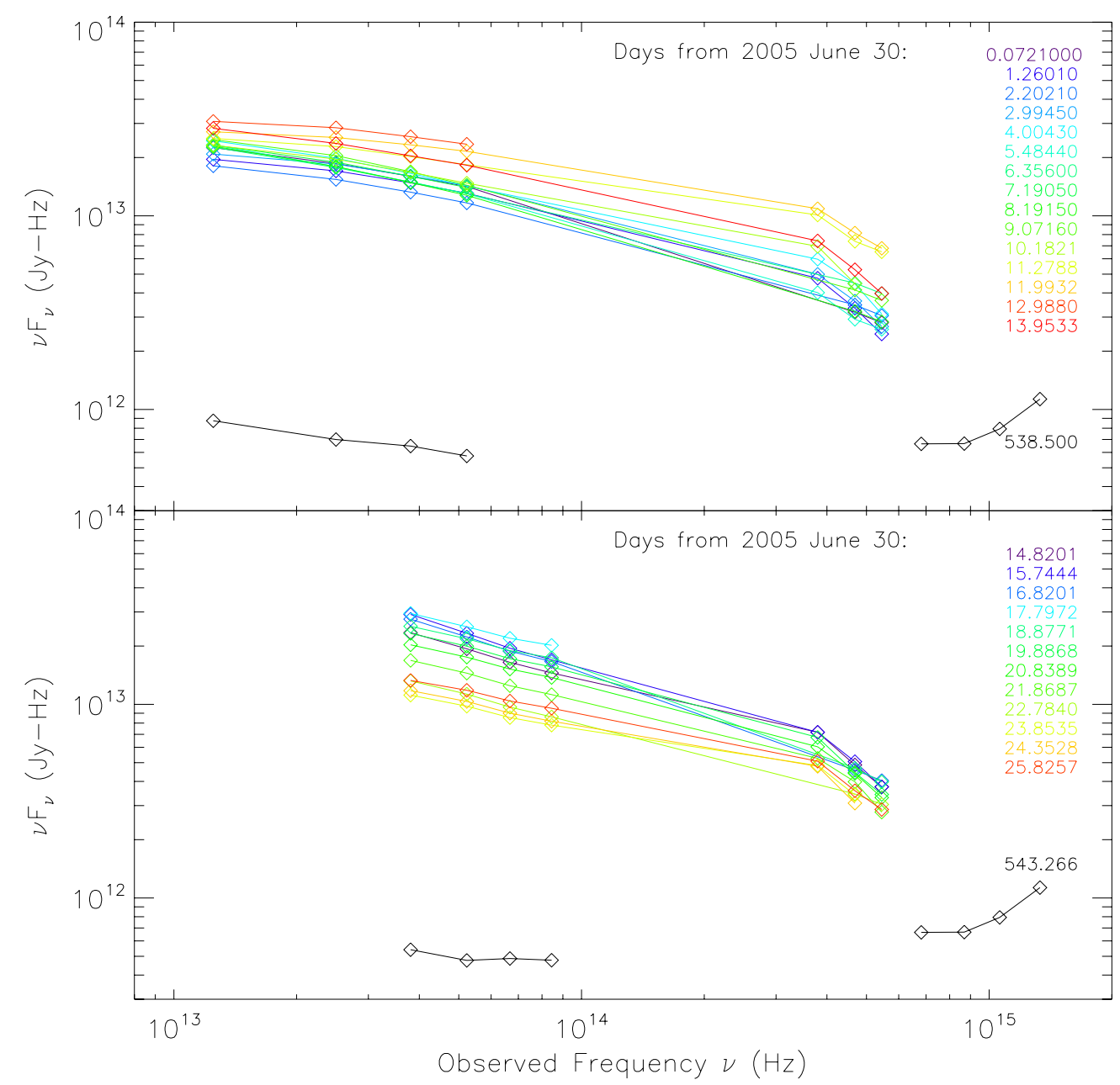

Figure 11. SED time series in mid-to-near-infrared and optical bands starting on 2005 June 30, and extending for 26 days, broken into two time periods including either the Spitzer IRS data or the Spitzer IRAC data, with dates distinguished in each frame by colors. Top: optical-to-infrared (IRS) SED variability during 2005 June-July and on 2006 December 20. Bottom: optical-to-infrared (IRAC) SED variability during 2005 June-July and on 2006 December 25. Most of the optical and near-infrared measurements in 2005 June-July are from Palomar and Foggy Bottom Observatories; a few points are taken from WEBT data provided by M. Villata (Villata et al. 2007). For comparison, we show the rise in the SED (at $\sim 10^{15} \mathrm{~Hz}$ ) attributed by Raiteri et al. (2008b) to the big blue bump; $B, U, W 1$, and $M 2$ data points are from the Optical Monitor on XMM, taken on 2006 December 18-19 (Table 3 of Raiteri et al. 2007).

Table 11

Representative Multiwavelength Spectrum in High State

\begin{tabular}{lcclcccc}
\hline \hline Year & Month & Day & \multicolumn{1}{c}{ MJD } & Band & $\begin{array}{c}\text { Freq } \\
(\mathrm{Hz})\end{array}$ & $\begin{array}{c}\text { Flux } \\
(\mathrm{Jy})\end{array}$ & $\begin{array}{c}\text { Error } \\
(\mathrm{Jy})\end{array}$ \\
\hline 2005 & 7 & 4 & 53555.555 & $221.5 \mathrm{GHz}$ & $2.215 \times 10^{11}$ & 41.0 & 2.0 \\
2005 & 7 & 5 & 53556.031 & $348.7 \mathrm{GHz}$ & $3.487 \times 10^{11}$ & 40.4 & 2.0 \\
2005 & 7 & 27 & 53578.6329211 & $160 \mu \mathrm{m}$ & $1.88 \times 10^{12}$ & 5.4 & 1.1 \\
2005 & 7 & 27 & 53578.6329211 & $70 \mu \mathrm{m}$ & $4.29 \times 10^{12}$ & 3.5 & 0.4 \\
2005 & 7 & 12 & 53563.9880 & $30 \mu \mathrm{m}$ & $1.00 \times 10^{13}$ & 2.95 & 0.10 \\
2007 & 7 & 27 & 53578.6329211 & $24 \mu \mathrm{m}$ & $1.25 \times 10^{13}$ & 1.12 & 0.11 \\
2005 & 7 & 12 & 53563.9880 & $24 \mu \mathrm{m}$ & $1.25 \times 10^{13}$ & 2.46 & 0.07 \\
2005 & 7 & 12 & 53563.9880 & $18 \mu \mathrm{m}$ & $1.67 \times 10^{13}$ & 1.80 & 0.05 \\
2005 & 7 & 12 & 53563.9880 & $12 \mu \mathrm{m}$ & $2.5 \times 10^{13}$ & 1.14 & 0.03 \\
2005 & 7 & 17 & 53569.29722 & $8.0 \mu \mathrm{m}$ & $3.75 \times 10^{13}$ & 0.77 & 0.04 \\
2005 & 7 & 12 & 53563.9880 & $6.0 \mu \mathrm{m}$ & $5.00 \times 10^{13}$ & 0.47 & 0.03 \\
2005 & 7 & 17 & 53569.29722 & $5.8 \mu \mathrm{m}$ & $5.17 \times 10^{13}$ & 0.48 & 0.02 \\
2005 & 7 & 17 & 53569.29722 & $4.5 \mu \mathrm{m}$ & $6.67 \times 10^{13}$ & 0.329 & 0.016 \\
2005 & 7 & 17 & 53569.29722 & $3.6 \mu \mathrm{m}$ & $8.33 \times 10^{13}$ & 0.239 & 0.012 \\
$2005^{\text {a }}$ & 7 & 23 & 53574.792 & $I$ & $3.72 \times 10^{14}$ & $1.14 \mathrm{E}-2$ & $0.02 \mathrm{E}-2$ \\
$2005^{\mathrm{a}}$ & 7 & 21 & 53572.8028 & $R$ & $4.56 \times 10^{14}$ & $8.54 \mathrm{E}-3$ & $0.09 \mathrm{E}-3$ \\
$2005^{\mathrm{a}}$ & 7 & 21 & 53572.8028 & $V$ & $5.44 \times 10^{14}$ & $6.50 \mathrm{E}-3$ & $0.06 \mathrm{E}-3$
\end{tabular}

Note. ${ }^{a}$ The $V$-, $R$-, and $I$-band photometry was corrected for Galactic absorption by factors of 1.39, 1.30, and 1.21, corresponding to magnitudes of $0.355,0.286$, and 0.208 , respectively. 


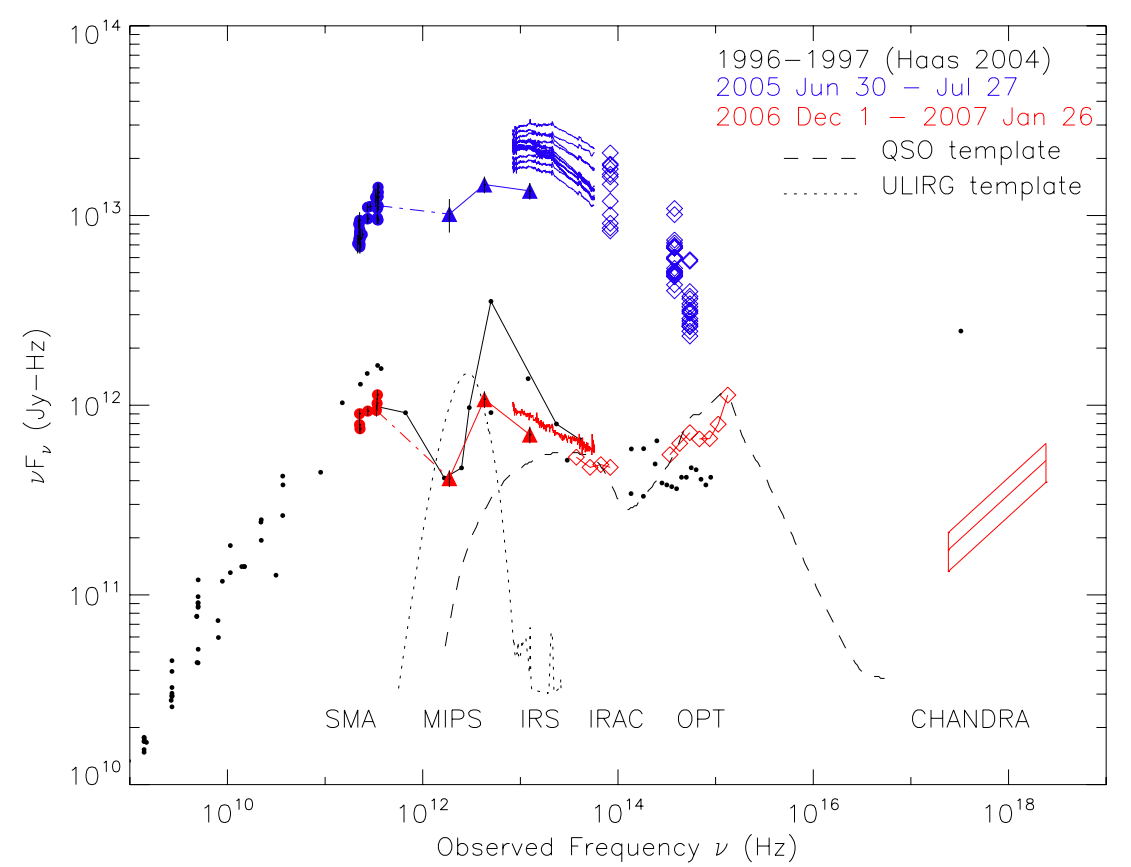

Figure 12. 3C 454.3 SED. Spitzer MIPS 160, 70, and $24 \mu \mathrm{m}$ photometry (triangles), IRS spectra (solid lines), and IRAC $3.6 \mu \mathrm{m}$ and optical photometry (diamonds) are plotted together with other multiwavelength data. The millimeter and sub-mm points are SMA calibration data. Optical photometry in 2005 is from Foggy Bottom Observatory and the automated Palomar $1.5 \mathrm{~m}$ telescope. XMM-OM (UVM2, UVW1, U, B) photometry for 2006 December 18-19 are from Raiteri et al. (2007, Table 3). V, $R$, and $I$ photometry for 2006 December 20 are from WEBT data provided by M. Villata (Raiteri et al. 2007; Villata et al. 2007). Both sets have been corrected for extinction. Chandra X-ray data for the 2007 minimum are indicated by the error box. The scattered small black dots are radio and optical photometry from the NED database, gathered from 1979-1995. The mean Richards et al. (2006) QSO SED (dashed line) and the Rieke et al. $10^{13} L_{\odot}$ ULIRG template (dotted line) are scaled and overplotted for comparison. Neither of these SEDs is compatible with the shape of the IR bump in the low-state 3C 454.3 SED, indicating a dominant nonthermal contribution from the jet. Note the presence of two synchrotron peaks, at IR and sub-mm wavelengths, in both the low and high states.

Table 12

Representative Multiwavelength Spectrum in Low State

\begin{tabular}{lccccccl}
\hline \hline Year & Month & Day & MJD & Band & $\begin{array}{c}\text { Freq } \\
(\mathrm{Hz})\end{array}$ & $\begin{array}{c}\text { Flux } \\
(\mathrm{Jy})\end{array}$ & $\begin{array}{c}\text { Error } \\
(\mathrm{Jy})\end{array}$ \\
\hline 2006 & 12 & 22 & 54091.309 & $225.5 \mathrm{GHz}$ & $2.255 \times 10^{11}$ & 4.0 & 0.2 \\
2006 & 12 & 29 & 54098.270 & $335.3 \mathrm{GHz}$ & $3.353 \times 10^{11}$ & 2.8 & 0.2 \\
2007 & 1 & 1 & 54101.9257388 & $160 \mu \mathrm{m}$ & $1.88 \times 10^{12}$ & 0.22 & 0.02 \\
2007 & 1 & 1 & 54101.9257388 & $70 \mu \mathrm{m}$ & $4.29 \times 10^{12}$ & 0.189 & 0.019 \\
2006 & 12 & 20 & 54089.5 & $30 \mu \mathrm{m}$ & $1.00 \times 10^{13}$ & 0.092 & 0.004 \\
2007 & 1 & 1 & 54101.9257388 & $24 \mu \mathrm{m}$ & $1.25 \times 10^{13}$ & 0.060 & 0.006 \\
2006 & 12 & 20 & 54089.5 & $24 \mu \mathrm{m}$ & $1.25 \times 10^{13}$ & 0.070 & 0.003 \\
2006 & 12 & 20 & 54089.5 & $18 \mu \mathrm{m}$ & $1.67 \times 10^{13}$ & 0.047 & 0.002 \\
2006 & 12 & 20 & 54089.5 & $12 \mu \mathrm{m}$ & $2.5 \times 10^{13}$ & 0.028 & 0.001 \\
2006 & 12 & 25 & 54081.76597 & $8.0 \mu \mathrm{m}$ & $3.75 \times 10^{13}$ & 0.014 & 0.007 \\
2006 & 12 & 20 & 54089.5 & $6.0 \mu \mathrm{m}$ & $5.00 \times 10^{13}$ & 0.012 & 0.001 \\
2006 & 12 & 25 & 54081.76597 & $5.8 \mu \mathrm{m}$ & $5.17 \times 10^{13}$ & $9.1 \mathrm{E}-3$ & $0.5 \mathrm{E}-3$ \\
2006 & 12 & 25 & 54081.76597 & $4.5 \mu \mathrm{m}$ & $6.67 \times 10^{13}$ & $7.3 \mathrm{E}-3$ & $0.4 \mathrm{E}-3$ \\
2006 & 12 & 25 & 54081.76597 & $3.6 \mu \mathrm{m}$ & $8.33 \times 10^{13}$ & $5.7 \mathrm{E}-3$ & $0.3 \mathrm{E}-3$ \\
$2006^{\mathrm{a}}$ & 12 & $18-19$ & 54088.5 & $B$ & $7.41 \times 10^{14}$ & $0.975 \mathrm{E}-3$ & $0.018 \mathrm{E}-3$ \\
$2006^{\mathrm{a}}$ & 12 & $18-19$ & 54088.5 & $U$ & $9.16 \times 10^{14}$ & $0.767 \mathrm{E}-3$ & $0.014 \mathrm{E}-3$ \\
$2006^{\mathrm{a}}$ & 12 & $18-19$ & 54088.5 & $U V W 1$ & $1.12 \times 10^{15}$ & $0.75 \mathrm{E}-3$ & $0.02 \mathrm{E}-3$ \\
$2006^{\mathrm{a}}$ & 12 & $18-19$ & 54088.5 & $U V M 2$ & $1.36 \times 10^{15}$ & $0.85 \mathrm{E}-3$ & $0.03 \mathrm{E}-3$ \\
$2007^{\mathrm{b}}$ & 1 & 1 & 54102.39907 & $2-10 \mathrm{keV}$ & $1.00 \times 10^{18}$ & $3.2 \mathrm{E}-7$ & $0.3 \mathrm{E}-7$ \\
\hline
\end{tabular}

Notes.

${ }^{a}$ Optical and ultraviolet data $(B, U, U V W 1, U V M 2)$ are from Raiteri et al. (2007), their Table 3. Some of the optical data used in this paper (not listed here), including data from 2006 December, are stored in the WEBT archive; for questions regarding their availability, please contact the WEBT President Massimo Villata (villata@oato.inaf.it)

${ }^{\mathrm{b}}$ Chandra $2-10 \mathrm{keV}$ flux was $5.8 \times 10^{-12} \mathrm{erg} \mathrm{cm}^{-2} \mathrm{~s}^{-1}$ with $\Gamma=1.53 \pm 0.12$.

In contrast, non-blazar quasars have two thermal peaks in their SEDs: an ultraviolet peak from the accretion disk and an infrared peak due to reprocessing of ultraviolet light by a dusty torus. We have plotted the mean (Richards et al. 2006) QSO
SED, compiled from Sloan Digital Sky Survey, Spitzer, nearIR, GALEX, Very Large Array, and ROSAT data in Figure 12, redshifted and scaled for comparison to the 3C 454.3 low state. The UV thermal peak is matched to the big and small blue 
bumps observed by XMM-OM (Raiteri et al. 2007). The IR bump in the low-state 3C 454.3 SED is significantly redder and peaks at a much lower frequency than the IR bump in the mean QSO SED. A thermal SED that peaks at $70 \mu \mathrm{m}$ is difficult to model with a standard dusty torus (e.g., Levenson et al. 2007), and would instead require that the QSO be deeply buried in an optically thick sphere of cold dust, contrary to the lack of any silicate absorption in the MIR spectrum and the presence of an unobscured big blue bump component in the UV SED.

We can also confidently rule out a significant thermal contribution from cold dust heated by star formation in the host galaxy of 3C 454.3. In Figure 12, we overplot the SED template constructed for a star formation dominated hyper-ultraluminous infrared galaxy (ULIRG) (Rieke et al. 2009) with bolometric luminosity $1 \times 10^{13} L_{\odot}$, scaled up by a factor of 2.2 to match the low-state $70 \mu \mathrm{m}$ (isotropic) luminosity of $2.9 \times 10^{46} \mathrm{erg} \mathrm{s}^{-1}$. Hyper-ULIRG galaxies are extremely rare in the universe and it would be very unusual for such a high-luminosity, radio-loud, type 1 quasar to have a starburst-dominated FIR SED. Furthermore, the low-state 5-70 $\mu \mathrm{m}$ power law with spectral index -1.3 (Section 3.2.5) is much flatter than the spectral index of the template hyper-ULIRG SED. We therefore conclude that the jet provides the dominant contribution to the low-state SED at $70 \mu \mathrm{m}$.

\subsection{Interpretation of the Two Synchrotron Peaks}

The presence of two synchrotron peaks has also been seen or suggested in at least three BL Lac objects, including BL Lacertae (Ravasio et al. 2003; Raiteri et al. 2009), Mrk 501 (Villata \& Raiteri 1999), and Mrk 421 (Donnarumma et al. $2009 b)$. The frequencies of the two peaks are in the near-IR to optical and far-UV to X-ray bands for BL Lac objects, in contrast to the sub-mm and far to mid-IR for the FSRQ 3C 454.3. Ravasio et al. (2003) suggest four possible explanations for the double peaks in BL Lacertae: (1) higher than normal ratio of optical to X-ray extinction, (2) bulk Comptonization, (3) Klein-Nishina (KN) effect on Compton cooling, and (4) two synchrotron emission regions. We can immediately rule out the first explanation for the two peaks in $3 \mathrm{C} 454.3$ because they occur at IR and lower frequencies, where extinction should not be significant.

Bulk Comptonization would require an external photon field with frequency $v_{\mathrm{ext}}=v_{p} / \Gamma_{j}^{2}=3 \times 10^{10}$ to $3 \times 10^{11} \mathrm{~Hz}$ for a jet with $\Gamma_{j}=12.3$ to produce the observed IR peak at $v_{p}=$ $4 \times 10^{12}$ to $3 \times 10^{13} \mathrm{~Hz}$. Coincidentally, the sub-mm synchrotron emission peak falls in this frequency range. However, this emission (particularly in the high state) is produced primarily by the relativistic jet, not an external source. We cannot rule out the possibility that there may be another, external source of synchrotron emission at that frequency providing seed photons for bulk Comptonization. A "cold" population of electrons may exist at the base of the jet where it has cooled from inverse Compton losses. However, bulk Comptonization of external optical-IR photons by this population would produce at feature at a few keV (Sikora et al. 2009), not in the IR.

While the KN effect can introduce a break in the slope at the high-energy end of the relativistic electron energy distribution and a corresponding break in the synchrotron emission spectrum (Moderski et al. 2005; Sikora et al. 2009, Figures 1 and 2) or even two synchrotron bumps in some cases (Dermer \& Atoyan 2002), it cannot produce the synchrotron peaks that we observe in 3C 454.3 (Figure 12). The frequency of the $\mathrm{KN}$ break should be at $4 \times 10^{16} B \Gamma_{j}^{-1}\left(v_{\text {ext }} / 3 \times 10^{14}\right)^{-2} \mathrm{~Hz}$ (Sikora et al. 2009,
Equation (54)), which would be at UV wavelengths ( $~ 900 \AA)$ for Comptonization of external IR photons from the dusty torus by a jet with $\Gamma_{j}=12.3$ and magnetic field of $B=1 \mathrm{G}$. This does not match the wavelength of the observed FIR minimum in the synchrotron SED.

We now focus on the most likely scenario of two synchrotron emission regions. Our observations of 3C 454.3 provide some clues to the relationship between the two (IR and sub-mm) synchrotron emission peaks. First, that both peaks occur in both the high and low states, over a period of six months (and also in the 1996-1997 ISO observations) indicates that the jet structures that produce them must persist in both the flaring and quiescent jet states. They are not a transient phenomenon particular to the 2005 jet flaring episode. However, further submm-IR monitoring at shorter timescales is crucial to elucidate the relationship between the two peaks. Second, the similar flux intensity (in $v F_{v}$ ) and factor of $\sim 40$ increase in the flux of both synchrotron peaks indicates that they come from regions or components in the near-side jet that have similar Doppler beaming factors.

The very dissimilar peak frequencies $\left(4 \times 10^{11}\right.$ versus $(4-40) \times 10^{12} \mathrm{~Hz}$ ), along with the lack of correlated variability at short timescales between the two peaks (Figure 10), indicate that they are produced in distinct regions of the jet with different physical parameters. The primary IR peak likely comes from a more compact region closer to the base of the jet than the sub-mm peak because of synchrotron self-absorption.

During the 2005 outburst, both regions appear to have responded to a major disturbance traveling along the jet. If the two regions are at different radii along the jet, then relativistic light travel time effects must shorten the apparent time between the disturbance at the first and second regions. The peak of the 2005 optical outburst occurred sometime before 2005 May 9 (the start of a WEBT observing campaign). The first peak of the $230 \mathrm{GHz}$ flare occurred sometime between 2005 May 16 and June 3, at $\Delta t \sim 7-25$ days after the peak of the optical outburst. Assuming the disturbance traveled down the jet at relativistic speed $v$, arriving first at the IR-optical emission region and second at the sub-mm emission region, then we estimate the distance between the two regions to be

$$
\Delta r=\frac{\beta c \Delta t /(1+z)}{1-\beta \cos \Theta_{o}},
$$

where $\beta=v / c=\left(1-1 / \Gamma_{j}^{2}\right)^{1 / 2}$ and $\Theta_{o}$ is the jet inclination to the line of sight. Assuming that the relativistic velocity and angle to the line of sight are the same as those measured for the associated superluminal radio knot K1 (Jorstad et al. 2010; $\left.\Gamma_{j}=12.3, \beta=0.99669, \Theta_{o}=1.0\right)$ gives $\Delta r=0.9-3$ pc. For comparison, the 0.068 mas angular size of the $43 \mathrm{GHz}$ VLBI core yields a jet width of $0.5 \mathrm{pc}$ and deprojected length of $30 \mathrm{pc}$, and the ejection site of the $\mathrm{K} 1 \mathrm{knot}$ is estimated to be $13 \pm 6 \mathrm{pc}$ downstream of the location of the optical flare (Jorstad et al. 2010).

We consider two possible geometries for the two synchrotron emission regions (Figure 13). The geometry suggested by Raiteri et al. (2009) for BL Lacertae is a helical jet, where the emission comes from two blobs moving at different angles to the line of sight, corresponding to different Doppler parameters. However, it is not clear in this picture why there would be two distinct synchrotron blobs rather than three or more blobs or a continuous distribution of emission along the helix. Rather, the existence of exactly two synchrotron peaks which are present 

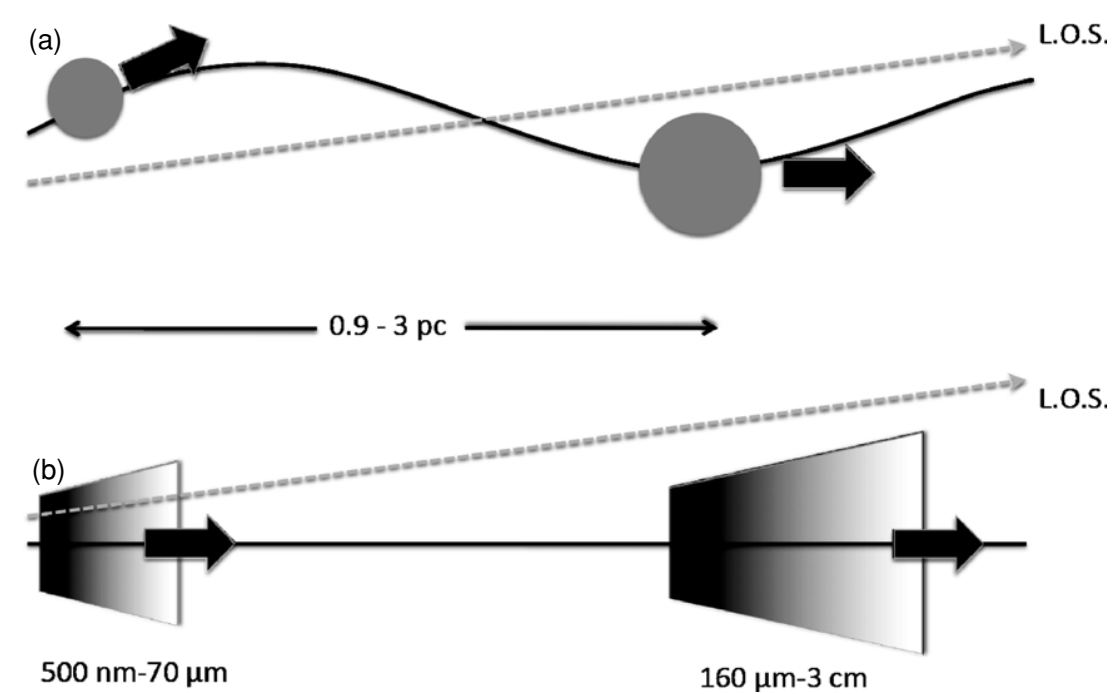

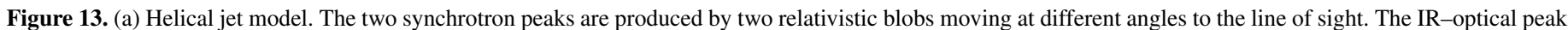

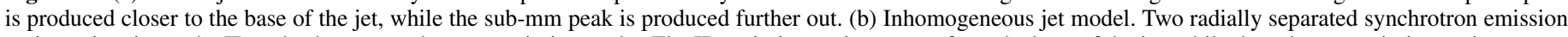

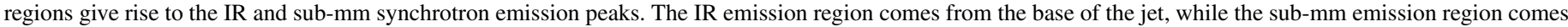
from a shock (possibly a jet recollimation shock) at a radius of $\sim 0.9-3$ pc. The direction to the observer's line of sight is marked "L.O.S."

in both the low and high states suggests a more permanent jet structure.

Another possible geometry for the two synchrotron emission peaks is that the IR peak comes from the base of the jet, where it becomes optically thin to IR photons, while the sub$\mathrm{mm}$ peak comes from a recollimation shock located further down the jet. Such a recollimation shock has been predicted with jet hydrodynamic simulations (Lind et al. 1989), where jet recollimation is caused by magnetic hoop stresses within the jet. This scenario has recently been invoked to explain the stationary (yet optically variable) HST 1 synchrotron emission knot observed in the jet of M87 (Nakamura et al. 2010). Alternatively, the sub-mm synchrotron emission region may mark a location where the jet runs into an external density enhancement such as the BLR.

Since two distinct synchrotron-emitting populations of particles are present, both populations would have to be folded into models that compute the contributions to the SED from synchrotron self-Compton emission, and EC emission from the accretion disk, BLR, and corona, as has been done for a single broken-power-law-distribution population by Vercellone et al. (2009). The resulting SEDs may show two peaks in the $\gamma$-ray band, corresponding to the two peaks in the infrared and sub-mm bands.

\subsection{The Origin of Mid-IR Flaring}

Our observations may be further interpreted in the framework of the relativistic shock model (Marscher \& Gear 1985). In this model, shocks are introduced at the base of a pre-existing relativistic jet by events in the accretion disk or supermassive black hole ergosphere. Shocks may be produced by an increase of the relativistic electron energy density, bulk flow speed, or changes in the magnetic field configuration. The electron density, energy density, and magnetic field will be enhanced at a shock front, leading to copious synchrotron and inverse Compton emission observed as a bright flare at radio-gamma frequencies.

According to the relativistic shock model, a peak in the SED comes from a location along the jet where the shock becomes optically thin to synchrotron self-absorption. As the shock region progresses along the jet and expands, it will become optically thin at progressively lower frequency, and the IR synchrotron peak will move from the mid-IR into the far-IR as it fades. The observed SED (Figure 12) does show this behavior, providing support for the relativistic shock model.

As the shock propagates down the jet, it loses energy primarily through inverse Compton scattering. An increase in the ambient photon density field, e.g., flares from the accretion disk or close encounters with broad-line clouds, would drain energy from the shock via Compton drag. This cannot explain mid-IR flaring, though it could certainly cause flaring of Compton X-rays and $\gamma$-rays, as for 3C279 (Wehrle et al. 1998).

The shock may be re-energized as it runs into inhomogeneities in the jet or surrounding interstellar medium (ISM), and bulk kinetic energy is converted to electron kinetic energy and magnetic fields. This could explain why we see large-amplitude mid-IR flaring with Spitzer two months after the peak of the 2005 May optical flare, after the original shock had propagated away from the energy source at the base of the jet.

Variability on longer timescales could also be caused by changes in the Doppler factor as the shock velocity changes its angle to the line of sight. As pointed out for BL Lacertae by Raiteri et al. (2009) and for 3C 454.3 by Villata et al. (2007), beaming in a curved jet varies along the jet and boosts the emission from various parts of the jet which are emitting at different frequencies, possibly causing changes in the observed flux and spectral index.

\subsection{Parameters for the IR-Optical Emission Region}

We use simple arguments to derive rough estimates of the physical parameters of the IR-optical flaring synchrotron emission regions in 2005 July. The source doubled its MIR (6 $\mu \mathrm{m})$ flux in $\Delta t_{\mathrm{IR}}=5$ days from 2005 July 9-14. The corresponding size of the synchrotron source in its rest frame is $c \Delta t_{\mathrm{IR}} \delta /(1+z)<0.05 \mathrm{pc}$, using a Doppler parameter of $\delta=23.4$ from Jorstad et al. (2010).

The magnetic field corresponding to the synchrotron frequency $v_{p}$ at the IR SED peak is $B=\left(2 \pi m_{e} c v_{p}\right)(1+z) /$ $\left(e \gamma^{2} \delta\right)=2.8 \times 10^{-3} v_{13} \gamma_{4}^{-2} \mathrm{G}$ (from Equation (1) of Burbidge et al. 1974). For the observed range of $v_{p}=4 \times$ 
$10^{12}$ to $4 \times 10^{13} \mathrm{~Hz}$ and a typical value of $\gamma \sim 10^{3}$, this gives $B=0.1-1 \mathrm{G}$. Assuming equipartition, the corresponding magnetic and electron energy densities are $U_{e}=U_{B}=B^{2} / 8 \pi=$ $5 \times 10^{-4}$ to $5 \times 10^{-2}$ erg $\mathrm{cm}^{-3}$.

The observed luminosity at the IR peak of the high-state SED is $v_{\text {obs }} L_{v, \text { obs }}=1.5 \times 10^{48} \mathrm{erg} \mathrm{s}{ }^{-1}$. In the rest frame of a spherically symmetric synchrotron emission region $L_{v}=$ $L_{v, \text { obs }} \delta^{-3}$ (Khembavi \& Narlikar 1999, from Equation (3.98)), so this corresponds to an isotropic luminosity of $v L_{v}=$ $v_{\mathrm{obs}} L_{v, \mathrm{obs}} \delta^{-4}=4 \times 10^{42} \mathrm{erg} \mathrm{s}^{-1}$. This is a small percentage $(\sim 0.008 \%)$ of the estimated jet kinetic luminosity $L_{\text {kin }}=$ $(4 / 3) \pi c r_{\text {jet }}^{2} \Gamma_{j}^{2} U_{e}(1+k) \leqslant 5 \times 10^{46} \mathrm{erg} \mathrm{s}^{-1}$, where we have used $r_{\text {jet }}<0.05 \mathrm{pc}$ for the MIR synchrotron source size and assumed an electron to proton energy ratio of $k=1.0$ in (Piner et al. 2003, Equation (9)).

The $e$-folding time for synchrotron cooling is $t_{\text {cool }}=$ $2.4 \times 10^{9} \gamma_{4}^{-1} B_{\mu \mathrm{G}}^{-2}$ year, where $B$ is in units of $\mu \mathrm{G}$, using Equation (9.23) of Krolik (1999). For $\gamma=10^{3}$ and $v_{p}=$ $4 \times 10^{13} \mathrm{~Hz}$ during the high state, $B=1 \mathrm{G}$ and the synchrotron cooling timescale is $t_{\text {cool }}=9$ days in the source frame ( $9 \mathrm{hr}$ in the observed frame for $\delta=23.4$ ). This value is consistent with variations on timescales of days and an upper limit of 1 day for the time delay between optical and infrared bands. The overall decline in optical flux on $e$-folding timescales of six months may be due to adiabatic expansion of the optically emitting jet component.

\section{CONCLUSIONS}

We observed 3C 454.3 with Spitzer IRS, IRAC, and MIPS, in a high state in 2005 June-July, 2 months after the 2005 May optical outburst, and with Spitzer and Chandra HETGs in a low state in 2006 December to 2007 January.

We find that:

1. There are no significant narrow or broad emission or absorption features in either the low- or high-resolution mid-IR spectra of the high state or low-resolution spectrum of the low state, consistent with a dominant nonthermal (synchrotron) emission mechanism during both states.

2. The mid-IR continuum emission is $\sim 30$ times brighter than during previous ISO observations. The mid-IR flux is highly variable, decreasing by a factor of 20-40 across the 5-35 $\mu \mathrm{m}$ band between 2005 July and 2006 December, indicating Doppler-boosted synchrotron emission from the jet.

3. There are two variable synchrotron emission peaks, one in the IR, and one in the sub-mm. The lack of correlated variability between the peaks on 1-30 day timescales and a lag of 7-25 days between the optical and sub-mm outbursts indicates two synchrotron emission regions, separated by $\sim 0.9-3$ pc.

4. The frequency of the primary synchrotron peak varies from $4 \times 10^{12} \mathrm{~Hz}$ to $4 \times 10^{13} \mathrm{~Hz}$, moving through the Spitzer MIPS and IRS bandpasses. The peak frequency is higher in outburst than during the low state, consistent with injection of high-energy electrons in a jet shock.

5. The 6-24 $\mu \mathrm{m}$ spectral slope becomes harder during flares and the peak of the SED moves to higher frequency. This may indicate that the shock is being re-energized as a new jet component runs into inhomogeneities in a slower, preexisting jet or the surrounding ISM.

6. No time delay between optical and infrared light curves was observed; any time delay is smaller than 0.5 days.
However, there are significant differences between the flaring behavior in the optical and infrared bands. Some flares have larger amplitude in the mid-IR, while others have larger amplitude in the optical.

7. The near-IR-optical spectral slope varies dramatically on the timescale of days, perhaps indicating injection of highenergy electrons in jet shocks.

This work is based on observations made with the Spitzer Space Telescope, which is operated by the Jet Propulsion Laboratory, California Institute of Technology under NASA contract 1407. Optical observations at Palomar Observatory were made possible through the NASA Space Interferometry Mission Preparatory Science Program for the Key Project on Active Galactic Nuclei. Support for this work was provided by NASA through awards issued by JPL/Caltech. The Submillimeter Array is a joint project between the Smithsonian Astrophysical Observatory and the Academia Sinica Institute of Astronomy and Astrophysics and is funded by the Smithsonian Institution and the Academia Sinica. This work is partly based on data taken and assembled by the WEBT collaboration and stored in the WEBT archive at the Osservatorio Astronomico di TorinoINAF (http://www.oato.inaf.it/blazars/webt/). We are grateful to Karl Gordon (Arizona) and Jason Surace (Spitzer Science Center) for assistance in processing MIPS and IRAC data, and to Booth Hartley for customizing IPAC Skyview for use on Intel Macs. We are grateful to Massimo Villata and Claudia Raiteri for providing WEBT data and for helpful discussions, and to Bill Keel (U. of Alabama) and Marco Chiaberge (STScI) for working with us on the Hubble images. We have made use of data from the NASA/IPAC Extragalactic Database (NED) which is operated by the Jet Propulsion Laboratory, California Institute of Technology, under contract with NASA.

\section{REFERENCES}

Abdo, A. A., Ackermann, M., Ajello, M., et al. 2009, ApJ, 699, 817 Ackermann, M., Ajello, M., Baldini, L., et al. 2010, ApJ, 721, 1383 Anderhub, H., Antonelli, L. A., Antoranz, P., et al. 2009, A\&A, 498, 83 Angione, R. J. 1971, AJ, 76, 412

Balonek, T. 2005a, vsnet-alert 8383

Balonek, T. 2005b, vsnet-alert 8405

Beckert, D. 1989, PASP, 101, 849

Bonning, E., Bailyn, C., Buxton, M., et al. 2010, ATel, 3022, 1

Bonning, E. W., Bailyn, C., Urry, C. M., et al. 2009, ApJ, 697, L81

Bonnoli, G., Ghisellini, G., Foschini, L., Tavecchio, F., \& Ghirlanda, G. 2011, MNRAS, 410, 368

Böttcher, M. 2010, in Fermi meets Jansky-AGN at Radio and Gamma-rays, ed. T. Savolainen, E. Ros, R. W. Porcas, \& J. A. Zensus, arXiv:1006.5048

Burbidge, G. R., Jones, T. W., \& O’Dell, S. L. 1974, ApJ, 193, 43

Cawthorne, T. V., \& Gabuzda, D. C. 1996, MNRAS, 278, 861

Cenko, S. B., Fox, D. B., Moon, D.-S., et al. 2006, PASP, 118, 1396

Cheung, C. C., Wardle, J. F. C., \& Chen, T. 2005, ApJ, 628, 104

Ciaramella, A., Bongardo, C., Aller, H. D., et al. 2004, A\&A, 419, 485

Cooper, N., Lister, M. L., \& Kochanczyk, M. D. 2007, ApJS, 171, 376

Craine, E. R. 1977, A Handbook of Quasistellar and BL Lacertae Objects (Astronomy and Astrophysics Series; Tucson, AZ: Pachart)

Decin, L., \& Eriksson, K. 2007, A\&A, 472, 1041

Decin, L., Morris, P. W., Appleton, P. N., et al. 2004, ApJS, 154, 408

Dermer, C., \& Atoyan, A. 2002, ApJ, 568, L81

Dickey, J. M., \& Lockman, F. J. 1990, ARA\&A, 28, 215

Donnarumma, I., Pucella, G., Vittorini, V., et al. 2009a, ApJ, 707, 1115

Donnarumma, I., Vittorini, V., Vercellone, S., et al. 2009b, ApJ, 691, L13

Finke, J. D., \& Dermer, C. D. 2010, ApJ, 714, 303

Fiorucci, M., Tosti, G., \& Rizzi, N. 1998, PASP, 110, 105

Foschini, L., Tagliaferri, G., Ghisellini, G., et al. 2010, MNRAS, 408, 448

Fossati, G., Maraschi, L., Celotti, A., Comastri, A., \& Ghisellini, G. 1998, MNRAS, 299, 433

Fuhrmann, L., Cucchiara, A., Marchili, N., et al. 2006, A\&A, 445, L1 
Ghisellini, G., Celotti, A., Fossati, G., Maraschi, L., \& Comastri, A. 1998, MNRAS, 301, 451

Ghisellini, G., \& Tavecchio, G. 2009, MNRAS, 397, 985

Giommi, P., Blustin, A. J., Capalbi, M., et al. 2006, A\&A, 456, 911

Gomez, J.-L., Marscher, A. P., \& Albierdi, A. 1999, ApJ, 577, 74

Gordon, K. D., Rieke, G. H., Engelbracht, C. W., et al. 2005, PASP, 117, 503

Gurwell, M. A., Peck, A. B., Hostler, S. R., Darrah, M. R., \& Katz, C. A. 2007, in ASP Conf. Ser. 375, From Z-Machines to ALMA: (Sub)Millimeter Spectroscopy of Galaxies, ed. A. J. Baker, J. Glenn, A. I. Harris, J. G. Mangum, \& M. S. Yun (San Francisco, CA: ASP), 234

Haas, M., Mller, S. A. H., Bertoldi, F., et al. 2004, A\&A, 424, 531

Hagen-Thorn, V. A., Efimova, N. V., Larionov, V. M., et al. 2009, Astrophys. Rep., 53, 510

Hartman, R. C., Bertsch, D. L., Dingus, B. L., et al. 1993, ApJ, 407, L41

Ho, P. T. P., Moran, J. M., \& Lo, K. Y. 2004, ApJ, 616, L1

Jorstad, S. G., Marscher, A. P., Larionov, V. M., et al. 2010, ApJ, 715, 362

Jorstad, S. G., Marscher, A. P., Lister, M. L., et al. 2005, AJ, 130, 1418

Jorstad, S. G., Marscher, A. P., Mattox, J. R., et al. 2001, ApJS, 134, 181

Kartaltepe, J. S., \& Balonek, T. J. 2007, AJ, 133, 2866

Kellerman, K. I., Lister, M. L., Homan, D. C., et al. 2004, ApJ, 609, 539

Khembavi, A. K., \& Narlikar, J. V. 1999, Quasars and Active Galactic Nuclei (Cambridge: Cambridge Univ. Press)

Krolik, J. 1999, Active Galactic Nuclei (Princeton, NJ: Princeton Univ. Press)

Lainela, M. 1994, A\&A, 286, 408

Larionov, V. M., Borisova, E., Jorstad, S. G., et al. 2010, ATel, 3003, 1

Levenson, N. A., Sirocky, M. M., Hao, L., et al. 2007, ApJ, 654, L45

Lind, K. R., Payne, D. G., Meier, D. L., \& Blandford, R. D. 1989, ApJ, 344, 89

Marscher, A. P., \& Gear, W. K. 1985, ApJ, 298, 114

Marscher, A. P., Jorstad, S. G., Mattox, J. R., \& Wehrle, A. E. 2002, ApJ, 577, 85

McNaron-Brown, K., Johnson, W. N., Jung, G. V., et al. 1995, ApJ, 451, 575

Moderski, R., Sikora, M., Coppi, P. S., \& Aharonian, F. 2005, MNRAS, 363, 954

Nakamura, M., Garafolo, D., \& Meier, D. L. 2010, ApJ, 721, 1783

Pacciani, L., Vittorini, V., Tavani, M., et al. 2010, ApJ, 716, L170

Pauliny-Toth, I. I. K., Porcas, R. W., Zensus, J. A., et al. 1987, Nature, 328, 778

Pearson, T. J., Readhead, A. C. S., \& Wilkinson, P. N. 1980, ApJ, 2236, 714
Pian, E., Falomo, R., \& Treves, A. 2005, MNRAS, 361, 919

Pian, E., Foschini, L., Beckmann, V., et al. 2006, A\&A, 449, L21

Piner, B. G., Unwin, S. C., Wehrle, A. E., et al. 2003, ApJ, 588, 716

Raiteri, C. M., Villata, M., Capetti, A., et al. 2009, A\&A, 507, 769

Raiteri, C. M., Villata, M., Chen, W. P., et al. 2008a, A\&A, 485, L17

Raiteri, C. M., Villata, M., Lanteri, L., et al. 1998, A\&AS, 130, 495

Raiteri, C. M., Villata, M., Larionov, V. M., et al. 2007, A\&A, 473, 819

Raiteri, C. M., Villata, M., Larionov, V. M., et al. 2008b, A\&A, 491, 755

Ravasio, M., Tagliaferri, G., Ghisellini, G., et al. 2003, A\&A, 408, 479

Reach, W. T., Megeath, S. T., Cohen, M., et al. 2005, PASP, 117, 978

Richards, G. T., Lacy, M., Storrie-Lombardi, L. J., et al. 2006, ApJS, 166, 470

Rieke, G. H., Alonso-Herrero, A., Weiner, B. J., et al. 2009, ApJ, 692, 556

Sasada, M., Uemura, M., Arai, A., et al. 2010, PASJ, 62, 645

Savolainen, T., Wiik, K., Valtaoja, E., Jorstad, S. G., \& Marscher, A. P. 2002, A\&A, 394, 851

Scott, W. K., Fomalont, E. B., Horiuchi, S., et al. 2004, ApJS, 155, 33

Sikora, M., Begelman, M. C., \& Rees, M. J. 1994, ApJ, 421, 153

Sikora, M., Stawarz, L., Moderski, R., Nalewajko, K., \& Madejski, G. M. 2009, ApJ, 704, 38

Smith, P. S., \& Balonek, T. J. 1998, PASP, 1110, 1164

Smith, P. S., Elston, R., Berriman, G., Allen, R. G., \& Balonek, T. J. 1988, ApJ, 326, L39

Smith, P. S., \& Wehrle, A. E. 2010, ATel, 2535, 1

Striani, E., Vercellone, S., Tavani, M., et al. 2010, ApJ, 718, 455

Tavecchio, F., Maraschi, L., Ghisellini, G., et al. 2002, ApJ, 575, 137

Vaughan, S., Edelson, R., Warwick, R. S., \& Uttley, P. 2003, MNRAS, 345, 1271

Vercellone, S., Chen, A. W., Giuliani, A., et al. 2008, ApJ, 676, L13

Vercellone, S., Chen, A. W., Vittorini, V., et al. 2009, ApJ, 690, 1018

Vercellone, S., D'Ammando, F., Vittorini, V., et al. 2010, ApJ, 712, 405

Villata, M., \& Raiteri, C. M. 1999, A\&A, 347, 30

Villata, M., Raiteri, C. M., Aller, M. F., et al. 2007, A\&A, 464, L5

Villata, M., Raiteri, C. M., Balonek, T. J., et al. 2006, A\&A, 453, 817

Villata, M., Raiteri, C. M., Gurwell, M. A., et al. 2009, A\&A, 504, L9

Wehrle, A. E., Pian, E., Urry, C. M., et al. 1998, ApJ, 497, 178

Zhang, S., Collmar, W., \& Schonfelder, V. 2005, A\&A, 444, 767 